\title{
Lifshitz black holes in IIA supergravity
}

\author{
Luke Barclay, ${ }^{a}$ Ruth Gregory, ${ }^{a, b}$ Susha Parameswaran, ${ }^{c}$ Gianmassimo Tasinato ${ }^{d}$ and \\ Ivonne Zavala ${ }^{e}$ \\ ${ }^{a}$ Centre for Particle Theory, \\ South Road, Durham, DH1 3LE, U.K. \\ ${ }^{b}$ Perimeter Institute, \\ 31 Caroline St, Waterloo, Ontario N2L 2Y5, Canada \\ ${ }^{c}$ Institute for Theoretical Physics, Leibniz University Hannover, \\ Welfengarten 1, 30167 Hannover, Germany \\ ${ }^{d}$ Institute of Cosmology \& Gravitation, University of Portsmouth, \\ PO1 3FX, U.K. \\ ${ }^{e}$ Centre for Theoretical Physics, University of Groningen, \\ Nijenborgh 4, 9747 AG Groningen, The Netherlands \\ E-mail: luke.barclay@durham.ac.uk, r.a.w.gregory@durham.ac.uk, \\ susha.parameswaran@itp.uni-hannover.de, \\ gianmassimo.tasinato@port.ac.uk, e.i.zavala@rug.nl
}

ABSTRACT: We compute string theoretic black hole solutions having Lifshitz asymptotics with a general dynamical exponent $z>1$. We start by constructing solutions in a flux compactification of six dimensional supergravity, then uplift them to massive type IIA supergravity. Alongside the Lifshitz black holes we study the simpler anti-de Sitter solutions, of which there are a 1-parameter family in this supergravity, and compare and contrast their properties. The black holes are characterized by a two-form and scalar charge, and we numerically explore their configuration space and thermodynamical aspects.

KEYwORDS: Gauge-gravity correspondence, Black Holes in String Theory, AdS-CFT Correspondence, Holography and condensed matter physics (AdS/CMT)

ARXIV EPRINT: 1203.0576 


\section{Contents}

1 Introduction $\quad 1$

2 The system 3

$\begin{array}{ll}2.1 \text { General planar spacetimes } & 6\end{array}$

3 Analytic results $\quad 7$

3.1 Anti-de Sitter solutions 9

$\begin{array}{lll}3.1 .1 \quad B \text {-charge } & 10\end{array}$

$\begin{array}{lll}3.1 .2 & \text { Scalar charge } & 11\end{array}$

$\begin{array}{ll}3.2 & \text { Lifshitz solutions } \\ \end{array}$

4 Numerical black hole solutions $\quad \mathbf{1 5}$

$\begin{array}{lll}4.1 & \text { AdS black holes } & 15\end{array}$

$\begin{array}{lll}4.2 & \text { Lifshitz black holes } & 17\end{array}$

4.3 Uplifting to type IIA in 10 dimensions 20

$\begin{array}{lll}5 & \text { Thermodynamics } & 21\end{array}$

6 Conclusions $\quad 24$

$\begin{array}{lr}\text { A The autonomous dynamical system } & 26\end{array}$

B Exact Lifshitz solutions $\quad 28$

B.1 4D LiBHs with constant dilaton 29

B.2 6D Romans' LiBH with constant dilaton? 30

\section{Introduction}

The use of holographic methods to explore strong coupling in gauge theories has yielded a particularly fruitful interaction between string theory and low temperature physics. The typical set-up uses the concept of gauge/gravity duality [1] in which a classical gravitational system with negative spacetime curvature has, on its boundary, equivalent degrees of freedom to a strongly coupled gauge theory. Temperature is gravitationally introduced into these systems by adding a black hole in the bulk spacetime, and different holographic dual theories can be constructed by having additional bulk fields (see $[2,3]$ for reviews of this approach).

The standard application of gauge/gravity duality is the adS/CFT correspondence, which yields a generally scale invariant boundary theory, however, more recently, attention 
has focussed on systems having more general scaling properties, such as non-relativistic field theories, $[4,5]$, or, pertinent to this investigation, a general dynamical Lifshitz scaling, $z$ :

$$
t \rightarrow \lambda^{z} t, \quad x^{i} \rightarrow \lambda x^{i}, \quad r \rightarrow r / \lambda .
$$

In order to produce such a dynamical scaling, the spacetime metric must be posited to have the following form

$$
d s^{2}=L^{2}\left(r^{2 z} d t^{2}-\frac{d r^{2}}{r^{2}}-r^{2} d x_{i} d x^{i}\right)
$$

which explicitly respects the scaling (1.1). In this metric, not only the asymptotics, but the full spacetime has the required scaling symmetry. Clearly, such a spacetime requires a matter content to produce this asymmetry, and this was first set out in the paper of Kachru et al. [6], in which charges and fluxes of topologically coupled gauge fields provided the necessary scaling. This theory is in fact on-shell equivalent to a somewhat simpler massive vector theory [7], although the $r \rightarrow 0$ singularity of these spacetimes exhibits certain pathologies $[8,9]$.

As with any holographic theory, although we can explore empirical simple models, in order to have confidence that there is indeed a holographically dual field theory we should be able to construct a qualitatively similar "top down" theory with Lifshitz scaling within string theory. After initial halted progress, string theory embeddings of Lifshitz geometries with dynamical exponent $z=2$ were found in [10-16], by making a consistent massive truncation of type IIB supergravity to a lower dimensional theory resembling the phenomenological construction of [6]. Soon after, a method for constructing Lifshitz spacetimes within string theory for arbitrary scaling exponent $z>1$ was put forward in [17]. In this approach, the Lifshitz space is constructed from a simple flux compactification of Romans' gauged supergravity in five and six dimensions, [18, 19], generalising the classic adS compactifications of those theories. The lower dimensional supergravity theories can be obtained by dimensionally reducing type IIA or IIB supergravity, as shown in [20-22], and any solutions can immediately be uplifted to ten dimensions. ${ }^{1}$ Further Lifshitz and AdS solutions in gauged supergravity and string theory have been also studied in [24].

In order to explore physical dualities, we need to be able to set our system at finite temperature, in other words, we need to introduce a black hole to our spacetime. Black holes in asymptotically Lifshitz spacetimes were initially hard to build. However by now several such solutions have been found in simple phenomenological models, starting with the numerical work of [25]. By engineering a matter or gravity content to source the desired geometry, some analytical solutions have also been constructed. Overall, Lifshitz black hole solutions in phenomenological models include numerical and analytic studies, fixed as well as arbitrary critical exponents, horizons with various topologies, extensions to other dimensions, higher-order theories of gravity, and Brans-Dicke models [26-44].

As to embedding Lifshitz black holes into string theory, this can now be done following on from the string constructions of pure Lifshitz geometries discussed above. Recently, numerical string Lifshitz black holes with dynamical exponent $z=2$ were presented in [45].

\footnotetext{
${ }^{1}$ See [23] for other examples of non-relativistic solutions in massive type IIA supergravity.
} 
As in [10-16], their method was to identify a consistent massive truncation from type IIB supergravity to a lower dimensional model resembling previous phenomenological constructions. In the present paper, we construct string Lifshitz black holes with general dynamical exponent $z>1$, generalising the lower dimensional supergravity/type IIA Lifshitz solutions, which were found in [17] by deforming adS solutions. Alongside the asymptotically Lifshitz black holes, we study related asymptotically adS black holes; thus we are able to draw on the intuition gleaned from the latter as well as identify which properties belong uniquely to the Lifshitz case.

With holographic condensed matter applications in mind, our interest is in planar black hole geometries, whose boundary field theory propagates in flat $2+1$ spacetime, and moreover we consider static geometries corresponding to equilibrium phases. Naturally, the black hole solutions are not so simple as their pure adS or Lifshitz cousins, with the exception of adS-Schwarzschild. By exciting the supergravity fields about this latter background in the probe limit, we learn about the charges inherent to our system. Further progress can be made by expressing the supergravity field equations as an autonomous dynamical system, whose fixed points are pure adS or Lifshitz. By perturbing close to the fixed points we can understand in detail how general interiors, including black holes, can flow to the adS/Lifshitz asymptotics. Indeed, this method allows us to analytically characterize all the possible asymptotic behaviours for static adS and Lifshitz black holes for our theory and moreover helps to numerically integrate to the full black hole solutions.

As might be expected, since our string/supergravity setup contains more degrees of freedom than the simple phenomenological models, the black holes have a rich structure. In the end, the black holes we find necessarily have some non-trivial scalar field, and aside from the horizon size, are characterized by two parameters, which can be interpreted as a form field charge and scalar charge. Thus we can begin to explore their configuration space, how the field profiles and thermodynamical properties change as the charges and dynamical exponent $z$ vary.

The paper is organised as follows. In the next section, we present the six dimensional supergravity theory where our black holes will be found, its pure adS and Lifshitz solutions and the general planar geometries that we will study. In section 3 we proceed to analyse this setup in detail, starting with some approximate analytic solutions, both through the whole spacetime but close to the adS-Schwarzchild black hole in the probe limit, and in the far field limit close to the asymptotic adS and Lifshitz geometry. We then build upon these results in section 4, to find numerical solutions describing adS and Lifshitz black holes, uplift them to type IIA supergravity, and study their behaviour. In section 5 we briefly discuss thermodynamical properties of the black holes and we conclude in section 6 . We give the details of the dynamical system we use to solve the supergravity field equations in appendix A. Finally, as an aside, in appendix B we identify some exact analytical black hole solutions in a generic dilatonic model, which could plausibly be related to a supergravity theory.

\section{The system}

In this section, we introduce the supergravity theory that will be the subject of the paper, present its pure adS and Lifshitz solutions, and propose the general Ansatz which we use to 
find planar black holes that asymptote adS and Lifshitz geometries. We consider six dimensional $\mathcal{N}=4$ gauged supergravity, first presented by Romans in [18]. This theory can be obtained from a consistent truncation of massive Type IIA supergravity, and thus the solutions of the six dimensional theory can be uplifted to solutions in string theory [20]. In [17] it was found that the field content and couplings of this theory admit Lifshitz solutions.

The bosonic field content of $6 \mathrm{D}$ Romans' supergravity consists of the metric, $g_{A B}$, a dilaton, $\phi$, an anti-symmetric two-form gauge field, $B_{A B}$, and a set of gauge vectors, $\left(A_{A}^{(i)}, \mathcal{A}_{A}\right)$ for the gauge group $\mathrm{SU}(2) \times \mathrm{U}(1)$. The bosonic part of the action for this theory is

$$
\begin{aligned}
S= & \int d^{6} x \sqrt{g_{6}}\left[-\frac{1}{4} R_{6}+\frac{1}{2}(\partial \phi)^{2}-\frac{e^{-\sqrt{2} \phi}}{4}\left(\mathcal{H}^{2}+F^{(i) 2}\right)+\frac{e^{2 \sqrt{2} \phi}}{12} G^{2}\right. \\
& -\frac{1}{8} \varepsilon^{A B C D E F} B_{A B}\left(\mathcal{F}_{C D} \mathcal{F}_{E F}+\mathrm{m} B_{C D} \mathcal{F}_{E F}+\frac{\mathrm{m}^{2}}{3} B_{C D} B_{E F}+F_{C D}^{(i)} F_{E F}^{(i)}\right) \\
& \left.+\frac{1}{8}\left(g^{2} e^{\sqrt{2} \phi}+4 g \mathrm{~m} e^{-\sqrt{2} \phi}-\mathrm{m}^{2} e^{-3 \sqrt{2} \phi}\right)\right]
\end{aligned}
$$

where $g$ is the gauge coupling, $\mathrm{m}$ is the mass of the 2 -form field $B_{A B}, \mathcal{F}_{A B}$ is a $\mathrm{U}(1)$ gauge field strength, $F_{A B}^{(i)}$ a nonabelian $\mathrm{SU}(2)$ gauge field strength, and $\mathcal{H}_{A B}=\mathcal{F}_{A B}+\mathrm{m} B_{A B}$. Spacetime indices $A, B, \ldots$ run from 0 to 5 , and $\varepsilon$ is the Levi-Civita tensor density. Notice the presence of Chern-Simons terms in the previous action, identified in [6] as an important ingredient for the existence Lifshitz configurations.

Varying the action gives the equations of motion:

$$
\begin{aligned}
R_{A B}= & 2 \partial_{A} \phi \partial_{B} \phi+\frac{1}{2} g_{A B} V(\phi)+e^{2 \sqrt{2} \phi}\left(G_{A}^{C D} G_{B C D}-\frac{1}{6} g_{A B} G^{2}\right) \\
& -e^{-\sqrt{2} \phi}\left(2 \mathcal{H}_{A}^{C} \mathcal{H}_{B C}+2 F_{A}^{i C} F_{B C}^{i}-\frac{1}{4} g_{A B}\left(\mathcal{H}^{2}+\left(F^{i}\right)^{2}\right)\right) \\
\square \phi= & \frac{1}{2} \frac{\partial V}{\partial \phi}+\frac{1}{3} \sqrt{\frac{1}{2}} e^{2 \sqrt{2} \phi} G^{2}+\frac{1}{2} \sqrt{\frac{1}{2}} e^{-\sqrt{2} \phi}\left(\mathcal{H}^{2}+\left(F^{(i)}\right)^{2}\right) \\
\nabla_{B}\left(e^{-\sqrt{2} \phi} \mathcal{H}^{B A}\right)= & \frac{1}{6} \epsilon^{A B C D E F} \mathcal{H}_{B C} G_{D E F} \\
\nabla_{B}\left(e^{-\sqrt{2} \phi} F^{(i) B A}\right)= & \frac{1}{6} \epsilon^{A B C D E F} F_{B C}^{(i)} G_{D E F} \\
\nabla_{C}\left(e^{2 \sqrt{2} \phi} G^{C A B}\right)= & -m e^{-\sqrt{2} \phi} \mathcal{H}^{A B}-\frac{1}{4} \epsilon^{A B C D E F}\left(\mathcal{H}_{C D} \mathcal{H}_{E F}+F_{C D}^{(i)} F_{E F}^{(i)}\right),
\end{aligned}
$$

where we have defined the scalar potential function:

$$
V(\phi)=\frac{1}{4}\left(g^{2} e^{\sqrt{2} \phi}+4 \mathrm{~m} g e^{-\sqrt{2} \phi}-\mathrm{m}^{2} e^{-3 \sqrt{2} \phi}\right) .
$$

Analogously to the Romans' solution $\operatorname{adS}_{4} \times H_{2}$ [18], it was shown in [17] that one can have a $\mathrm{Li}_{4} \times H_{2}$ dimensional reduction of this $6 \mathrm{D}$ supergravity to a $4 \mathrm{D}$ Lifshitz space with an internal hyperbolic manifold threaded by non-abelian magnetic flux. The solution 
is given by

$$
d s^{2}=L^{2}\left(r^{2 z} d t^{2}-r^{2} d x_{1}^{2}-r^{2} d x_{2}^{2}-\frac{d r^{2}}{r^{2}}\right)-\frac{a^{2}}{y_{2}^{2}}\left(d y_{1}^{2}+d y_{2}^{2}\right)
$$

where $a$ is a constant, the radius of curvature of the hyperboloid which can be taken to be compact (see [46] for details). The dilaton is also chosen to be constant, $\phi=\phi_{0}$, and the field configurations are

$$
\begin{aligned}
& F_{t r}^{(3)}=q b L^{3} e^{\sqrt{2} \phi_{0}} r^{z-1} \quad F_{y_{1} y_{2}}^{(3)}=\frac{q}{y_{2}^{2}} \\
& G_{x_{1} x_{2} r}=b L^{3} r \quad \Rightarrow \quad B_{x_{1} x_{2}}=\frac{b}{2} L^{3} r^{2} .
\end{aligned}
$$

The relations between the various constants are somewhat simplified by performing the following rescalings

$$
\begin{array}{lll}
\hat{b} & =L b e^{\sqrt{2} \phi_{0}} & \hat{q}=L e^{-\phi_{0} / \sqrt{2}} q / a^{2} \\
\hat{g}=L g e^{\phi_{0} / \sqrt{2}} & \hat{a}=a / L & \hat{m}=L \mathrm{~m} e^{-3 \phi_{0} / \sqrt{2}} .
\end{array}
$$

Equations (2.2) to (2.6) then reduce to a simple set of algebraic equations with the following general solutions

$$
\begin{array}{lll}
\hat{b}^{2}=z-1 & \hat{g}^{2}=2 z(4+z) & \frac{\hat{m}^{2}}{2}=\frac{6+z \mp 2 \sqrt{2(z+4)}}{z} \\
\hat{q}^{2}=\frac{(2+z)(z-3) \pm 2 \sqrt{2(z+4)}}{2 z} & \frac{1}{\hat{a}^{2}}=6+3 z \mp 2 \sqrt{2(z+4)}
\end{array}
$$

These define two families of Lifshitz spacetimes, one for each branch of the square root in (2.11). The requirement that $\hat{b}$ is real restricts $z$ to $z \geq 1$ and for the lower sign choice, for $\hat{q}$ to be real one finds that $z$ must be greater than approximately 4.29 .

In addition to these Lifshitz solutions, the system also allows for an independent one parameter family of adS solutions when $z=1$ and $\hat{b}=0$. These solutions can be parametrized by either $\hat{g}$ or $\hat{m}$, the latter case giving

$$
\hat{g}=\frac{\hat{m}^{2}+6}{2 \hat{m}}, \quad \hat{q}^{2}=-\frac{5 \hat{m}^{4}-36 \hat{m}^{2}+36}{16 \hat{m}^{2}}, \quad \frac{1}{\hat{a}^{2}}=\frac{5 \hat{m}^{4}-12 \hat{m}^{2}+36}{8 \hat{m}^{2}} .
$$

In this case the requirement that $\hat{q}$ is real implies that $\hat{m} \in\left[\sqrt{\frac{6}{5}}, \sqrt{6}\right]$. When $\hat{m}=\sqrt{10}-2$ the adS and (upper sign) Lifshitz solutions touch at $z=1$. Figure 1 represents the values of $z$ and $\hat{m}$ that these Lifshitz and adS solutions can take.

In what follows, we continue to analyze adS and Lifshitz solutions, generalizing the previous discussion to the case of asymptotically adS and Lifshitz space-times. Insights acquired when analyzing asymptotically adS configurations will be of great help when considering asymptotically Lifshitz space-times. 


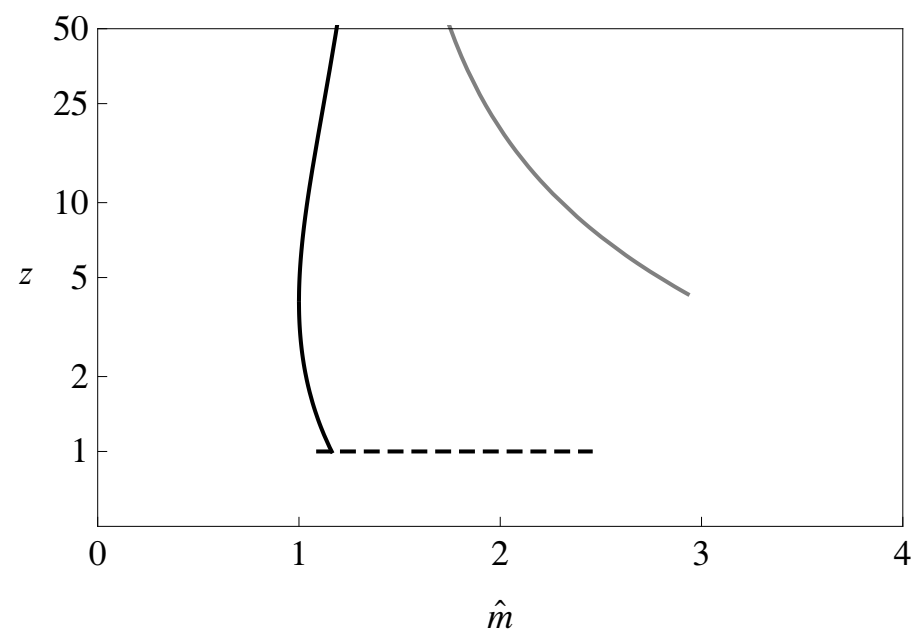

Figure 1. Plot showing the values of $z$ and $\hat{m}$ that the Lifshitz and adS solutions can take. The horizontal dashed line indicates the adS solutions, with $z=1$. The black line corresponds to the Lifshitz solutions with the upper sign choice in (2.11) and the grey to the lower sign choice. Notice that adS and Lifshitz solutions meet at $\hat{m}=\sqrt{10}-2$.

\subsection{General planar spacetimes}

Our main aim is to characterize asymptotically adS and Lifshitz black hole solutions. We look for solutions which respect the planar symmetry and static nature of the metric (2.8), meaning the alterations need only have radial dependence, $\phi=\phi(r)$ and

$$
d s^{2}=L^{2}\left[e^{2 f(r)} d t^{2}-e^{2 c(r)} d \mathbf{x}^{2}-e^{2 d(r)} d r^{2}\right]-e^{2 h(r)} d H_{2}^{2}
$$

We choose our field strength Ansätze to be

$$
F_{t r}^{(3)}=L^{2} Q(r), \quad F_{y_{1} y_{2}}^{(3)}=\frac{q}{y_{2}^{2}}, \quad B_{x_{1} x_{2}}=\frac{L^{2}}{2} e^{-\sqrt{2} \phi_{0}} P(r),
$$

which gives the gauge equations:

$$
\begin{gathered}
\left(e^{2 c+2 h-f-d-\sqrt{2} \phi} Q\right)^{\prime}=q e^{-\sqrt{2} \phi_{0}} P^{\prime} \\
\left(e^{f-d+2 h-2 c+2 \sqrt{2} \phi} e^{-\sqrt{2} \phi_{0}} P^{\prime}\right)^{\prime}=m^{2} L^{2} e^{-\sqrt{2} \phi_{0}} P e^{f+d-2 c+2 h-\sqrt{2} \phi}+4 L^{2} q Q .
\end{gathered}
$$

Integrating (2.15) and noting that $Q \rightarrow 0$ as $P \rightarrow 0$ from (2.16), we obtain:

$$
Q(r)=e^{\sqrt{2} \phi(r)+f(r)+d(r)-2 c(r)-2 h(r)} q e^{-\sqrt{2} \phi_{0}} P(r),
$$

hence there is a single equation of motion for the gauge fields:

$$
\left(e^{f-d+2 h-2 c+2 \sqrt{2} \phi} P^{\prime}\right)^{\prime}=L^{2} P e^{f+d-2 c+2 h-\sqrt{2} \phi}\left(m^{2}+4 q^{2} e^{2 \sqrt{2} \phi-4 h}\right) .
$$


The remaining equations are:

$$
\begin{aligned}
\frac{\sqrt{2}}{\sqrt{g}}\left(\frac{\sqrt{g} \phi^{\prime}}{L^{2} e^{2 d}}\right)^{\prime}= & P^{2} e^{-2 \sqrt{2} \phi_{0}-\sqrt{2} \phi-4 c}\left(q^{2} e^{2 \sqrt{2} \phi-4 h}-\frac{\mathrm{m}^{2}}{4}\right)+\frac{P^{\prime 2}}{2 L^{2}} e^{2 \sqrt{2}\left(\phi-\phi_{0}\right)-4 c-2 d} \\
& -q^{2} e^{-\sqrt{2} \phi-4 h}-\frac{1}{4}\left(g^{2} e^{\sqrt{2} \phi}-4 \mathrm{~m} g e^{-\sqrt{2} \phi}+3 \mathrm{~m}^{2} e^{-3 \sqrt{2} \phi}\right) \\
\frac{2}{\sqrt{g}}\left(\frac{\sqrt{g} f^{\prime}}{L^{2} e^{2 d}}\right)^{\prime}= & P^{2} e^{-2 \sqrt{2} \phi_{0}-\sqrt{2} \phi-4 c}\left(3 q^{2} e^{2 \sqrt{2} \phi-4 h}+\frac{\mathrm{m}^{2}}{4}\right)+\frac{P^{\prime 2}}{2 L^{2}} e^{2 \sqrt{2}\left(\phi-\phi_{0}\right)-4 c-2 d} \\
& +q^{2} e^{-\sqrt{2} \phi-4 h}+\frac{1}{4}\left(g^{2} e^{\sqrt{2} \phi}+4 \mathrm{~m} g e^{-\sqrt{2} \phi}-\mathrm{m}^{2} e^{-3 \sqrt{2} \phi}\right) \\
\frac{2}{\sqrt{g}}\left(\frac{\sqrt{g} c^{\prime}}{L^{2} e^{2 d}}\right)^{\prime}= & P^{2} e^{-2 \sqrt{2} \phi_{0}-\sqrt{2} \phi-4 c}\left(-q^{2} e^{2 \sqrt{2} \phi-4 h}-\frac{3 \mathrm{~m}^{2}}{4}\right)-\frac{P^{\prime 2}}{2 L^{2}} e^{2 \sqrt{2}\left(\phi-\phi_{0}\right)-4 c-2 d} \\
& +q^{2} e^{-\sqrt{2} \phi-4 h}+\frac{1}{4}\left(g^{2} e^{\sqrt{2} \phi}+4 \mathrm{~m} g e^{-\sqrt{2} \phi}-\mathrm{m}^{2} e^{-3 \sqrt{2} \phi}\right) \\
\frac{2}{\sqrt{g}}\left(\frac{\sqrt{g} h^{\prime}}{L^{2} e^{2 d}}\right)^{\prime}= & P^{2} e^{-2 \sqrt{2} \phi_{0}-\sqrt{2} \phi-4 c}\left(-q^{2} e^{2 \sqrt{2} \phi-4 h}+\frac{\mathrm{m}^{2}}{4}\right)+\frac{P^{\prime 2}}{2 L^{2}} e^{2 \sqrt{2}\left(\phi-\phi_{0}\right)-4 c-2 d} \\
& -3 q^{2} e^{-\sqrt{2} \phi-4 h}+\frac{1}{4}\left(g^{2} e^{\sqrt{2} \phi}+4 \mathrm{~m} g e^{-\sqrt{2} \phi}-\mathrm{m}^{2} e^{-3 \sqrt{2} \phi}\right)-2 e^{-2 h}
\end{aligned}
$$

together with the first integral of the Einstein equations:

$$
\begin{aligned}
& 2 f^{\prime} c^{\prime}+2 f^{\prime} h^{\prime}+4 c^{\prime} h^{\prime}+c^{\prime 2}+h^{\prime 2}-\phi^{\prime 2}-\frac{P^{\prime 2}}{4} e^{2 \sqrt{2}\left(\phi-\phi_{0}\right)-4 c}= \\
& L^{2} e^{2 d}\left[-e^{-2 h}-q^{2} e^{-\sqrt{2} \phi-4 h}-P^{2} e^{-2 \sqrt{2} \phi_{0}-\sqrt{2} \phi-4 c}\left(q^{2} e^{2 \sqrt{2} \phi-4 h}+\frac{\mathrm{m}^{2}}{4}\right)+V(\phi)\right] .
\end{aligned}
$$

In analysing the solutions of these equations, it is particularly useful to consider the equations of motion from a dynamical systems perspective. The exact Lifshitz or adS geometries correspond to fixed points of the dynamical system, and a perturbative analysis around the fixed points indicates the flows of general interior solutions to the asymptotic Lifshitz or adS geometry. This general methodology was used in [47] to explore flows between exact Lifshitz and adS solutions.

Although the system (2.18)-(2.22) appears to be nine dimensional, there is a redundant gauge degree of freedom corresponding to a rescaling of the coordinates, and also the Bianchi identity (2.23), which reduces the order of the system to seven. Perturbing around a fixed point solution therefore will give a seven dimensional solution space (some of which will correspond to unphysical singular solutions), spanned by the eigenvectors of the perturbation operator around the critical point, with a radial fall-off given by the corresponding eigenvalue $\Delta$. In appendix A we provide the details of a dynamical systems analysis of these equations of motion, and the derivation of the eigenvalues. The eigenvalues will provide crucial information about the physical charges that characterise a given solution.

\section{Analytic results}

In order to develop a general understanding that will be later used to determine numerical black holes configurations, it is useful to analytically explore the allowed asymptotic 
behaviour of adS and Lifshitz solutions for our theory. In this section, by implementing the dynamical system analysis developed in appendix A, we will first identify perturbative solutions which describe flows towards an adS boundary at large $r$. Among these is the exact adS-Schwarzschild solution, and we analyse linearized solutions about this background, throughout the space-time from horizon to boundary. We then study flows towards a Lifshitz boundary at large $r$, for arbitrary dynamical exponent $z$. In this way, we are also able to observe how asymptotically Lifshitz geometries for $z>1$ reduce to asymptotically adS geometries at $z=1$.

For convenience, we choose the radial coordinate to correspond to the area gauge as in (1.2), i.e. $c(r)=\log r$, and rewrite our metric and scalar functions in terms of deviations from the known Lifshitz background:

$$
\begin{array}{lll}
\phi(r)=\phi_{0}+\varphi(r) / \sqrt{2}, & h(r)=\log a+\frac{1}{2} \ln H(r), & P(r)=r^{2} p(r) \\
f(r)=z \ln r+\frac{1}{2} \ln F(r), & d(r)=-\ln r-\frac{1}{2} \ln D(r) . &
\end{array}
$$

This gives us the field equations

$$
\begin{aligned}
\frac{\left(r^{z+3} \sqrt{F D} H \varphi^{\prime}\right)^{\prime}}{r^{z+1} H \sqrt{F / D}}= & -\frac{\hat{q}^{2} e^{-\varphi}}{H^{2}}+p^{2}\left(\frac{\hat{q}^{2} e^{\varphi}}{H^{2}}-\frac{\hat{m}^{2} e^{-\varphi}}{4}\right)+2 D e^{2 \varphi}\left(p+\frac{r p^{\prime}}{2}\right)^{2}-\frac{\partial \hat{V}}{\partial \varphi} \\
\frac{\left(r^{z+3} \sqrt{F D} H^{\prime}\right)^{\prime}}{r^{z+1} H \sqrt{F / D}=} & -3 \frac{\hat{q}^{2} e^{-\varphi}}{H^{2}}-p^{2}\left(\frac{\hat{q}^{2} e^{\varphi}}{H^{2}}-\frac{\hat{m}^{2} e^{-\varphi}}{4}\right)+2 D e^{2 \varphi}\left(p+\frac{r p^{\prime}}{2}\right)^{2} \\
& +\hat{V}(\varphi)-\frac{2}{\hat{a}^{2} H} \\
\frac{\left(r^{z+3} \sqrt{D / F} H F^{\prime}\right)^{\prime}}{r^{z+1} H \sqrt{F / D}=} & (1-z) \frac{\hat{q}^{2} e^{-\varphi}}{H^{2}}+p^{2}\left(\frac{(3+z) \hat{q}^{2} e^{\varphi}}{H^{2}}+\frac{(1+3 z) \hat{m}^{2} e^{-\varphi}}{4}\right) \\
& +2(1+z) D e^{2 \varphi}\left(p+\frac{r p^{\prime}}{2}\right)^{2}+(1-z) \hat{V}(\varphi) \\
\frac{\left(2 r^{z+2} H \sqrt{F D}\right)^{\prime}}{r^{z+1} H \sqrt{F / D}}= & \frac{\hat{q}^{2} e^{-\varphi}}{H^{2}}-p^{2}\left(\frac{\hat{q}^{2} e^{\varphi}}{H^{2}}+\frac{3 \hat{m}^{2} e^{-\varphi}}{4}\right)-2 D e^{2 \varphi}\left(p+\frac{r p^{\prime}}{2}\right)^{2}+\hat{V}(\varphi),
\end{aligned}
$$

together with the gauge equation

$$
\left(r^{z} \sqrt{F D} e^{2 \varphi} H\left(2 p+r p^{\prime}\right)\right)^{\prime}=r^{z-1} p H e^{-\varphi} \sqrt{F / D}\left(\hat{m}^{2}+\frac{4 \hat{q}^{2} e^{2 \varphi}}{H^{2}}\right),
$$

and the first integral

$$
\begin{aligned}
& D\left[2 z+1+\frac{r F^{\prime}}{F}+(z+2) \frac{r H^{\prime}}{H}+\frac{r^{2} H^{\prime} F^{\prime}}{2 H F}+\frac{r^{2} H^{\prime 2}}{4 H^{2}}-\frac{r^{2} \varphi^{\prime 2}}{2}-\left(p+\frac{r p^{\prime}}{2}\right)^{2} e^{2 \varphi}\right] \\
& \quad=\hat{V}(\varphi)-\frac{1}{\hat{a}^{2} H}-\frac{\hat{q}^{2} e^{-\varphi}}{H^{2}}-p^{2}\left(\frac{\hat{q}^{2} e^{\varphi}}{H^{2}}+\frac{\hat{m}^{2} e^{-\varphi}}{4}\right),
\end{aligned}
$$

where

$$
\hat{V}(\varphi)=\frac{1}{4}\left(\hat{g}^{2} e^{\varphi}+4 \hat{m} \hat{g} e^{-\varphi}-\hat{m}^{2} e^{-3 \varphi}\right) .
$$




\subsection{Anti-de Sitter solutions}

We start with a detailed analysis of the adS branch of solutions to our system. These configurations are easier to study than Lifshitz space-times, yet they give insights into what charges are inherent in the system, and what aspects of black hole solutions are uniquely Lifshitz. In the adS case, of course, we already know an analytic black hole, the adS-Schwarzschild solution:

$$
H=1, \quad \varphi=p=0, \quad F=D=1-\left(\frac{r_{+}}{r}\right)^{3} .
$$

From the point of view of the dynamical system discussed in appendix A, this corresponds to the nonlinear evolution into the interior of the asymptotic eigenvalue ${ }^{2} \Delta=-3$ (the $1 / r^{3}$ fall-off), which has an eigenvector with components only in the directions corresponding to deformations of the $4 \mathrm{D}$ geometry, as in eq. (3.9).

It is useful however to continue with a more general analysis of perturbative solutions which asymptote adS, as this will enable a more direct comparison with the Lifshitz case. A general analysis of the perturbations around the adS fixed point (see appendix A) yields the eigenvalues plotted in figure 2. As explained in detail in the figure caption, these eigenvalues give the exponents of $r$ in the asymptotic solutions for the various fields, as function of $\hat{m}$. Each field has a fall-off of a pair of exponents which are symmetric about $-3 / 2$, and whose coefficients can be interpreted as a source and operator in the boundary field theory. The pure 'black hole' mode, $\Delta=-3$ is in this sense dual to the zero mode which takes us along the parameter space of adS solutions.

A combination of the dotted and dashed black and grey lines in figure 2 corresponds to switching on the dilaton and breather modes, keeping $P$ equal to zero. The solid black lines in the figure are associated with the turning on of the 2 -form charge $P$ only. At $\hat{m}=\sqrt{10}-2$, the value of $\hat{m}$ for which the pure adS and Lifshitz solutions coincide (since $z=1$ ), and the charge and mass deformations of adS become degenerate. Since the Pequation decouples at leading order, it is not difficult to extract the charged perturbations of adS:

$$
H=F=D=1, \quad \varphi=0, \quad p=p_{0}-\frac{p_{3}}{r^{3}} .
$$

The $p_{0}$ deformation corresponds to the zero mode (the upper solid black line at $\hat{m}=\sqrt{10}-2$ in figure 2) which moves the solution onto the Lifshitz branch whereas the $p_{3}$ deformation identifies the pure charge eigenvector.

As we learn in what follows, perturbations of asymptotically Lifshitz configurations have different eigenvectors and eigenvalues, conveniently parameterized by the quantity $z$. On the other hand, when $\hat{m}=\sqrt{10}-2$ (corresponding to $z=1$ ) the Lifshitz deformations and adS deformations should coincide, as there is a degeneracy of the eigensystem at this point: the corresponding Lifshitz deformations are a combination of the pure mass and pure charge asymptotically adS solutions. In section 3.2 we analyze the corresponding Lifshitz asymptotic solutions and how they join the adS ones at $z=1$.

\footnotetext{
${ }^{2}$ Asymptotic eigenvalues $\Delta_{i}$ control the fall-off $r^{-\Delta_{i}}$ of a given function at large $r$.
} 


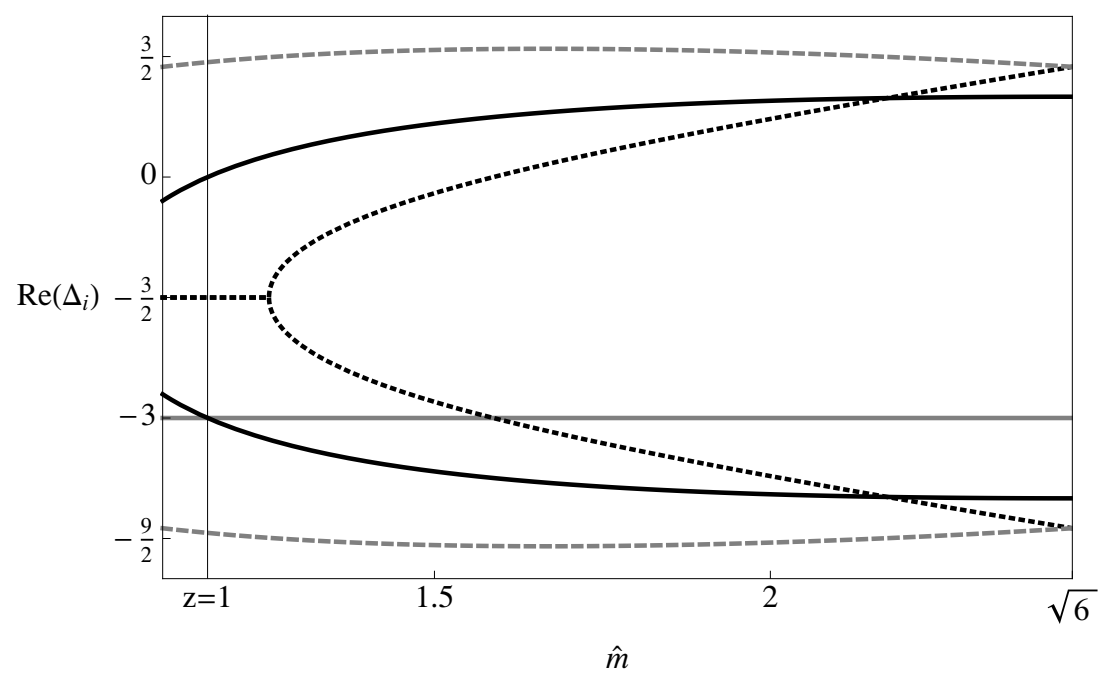

Figure 2. Plot showing the real part of the field exponents as they asymptotically approach an adS space-time, as a function of the parameter $\hat{m}$. Each pair of exponents sums to -3 . The solid black lines correspond to switching on a B-charge in the interior spacetime, and the solid horizontal grey line is the black hole solution. A combination of the dotted and dashed black and grey lines corresponds to switching on the dilaton and breather modes. (The joining of the dotted black lines for small $\hat{m}$ indicates that the exponents turn complex.) Notice that at $\hat{m}=\sqrt{10}-2$, which is the value of $\hat{m}$ at which the adS fixed point solution is equivalent to a $z=1$ upper branch Lifshitz solution, there is a degeneracy in the eigenvalues: the mass and charge deformations have the same fall-off.

Turning now to black hole solutions, we next explore linearized solutions for scalar and gauge charges around the known adS-Schwarzschild black hole background of eq. (3.9). We stress that the eigenvalue analysis discussed above refers to perturbations of the full system around the adS background, and as such describe how the geometry and fields asymptote the adS boundary at large $r$. In contrast, in the next two subsections we seek linearized solutions of either the $B$-field, or the dilaton and breather mode, around the black hole background. These, at leading order, do not include perturbations of the black hole geometry, but they are solutions of the scalar or gauge fields for the full range of the space-time from the horizon to the boundary. Our linearized solutions, therefore, should asymptote one or more of the eigenvalue solutions near infinity, at least for the fields we are perturbing. On the other hand, they are more informative since they provide some further understanding on how field configurations behave in the black hole background. Our aim here is to identify the eigensolutions of the full system, so as to have a physical interpretation of the various eigenvectors which we can then use to understand the Lifshitz system.

\subsubsection{B-charge}

We start by allowing $p$ to vary, but keeping $\varphi$ and $H$ constant over the black hole background of eq. (3.9). Since $P$ is related to the electric field and the flux of the $B_{A B}$ field of the system we shall refer to these solutions as charged black holes. Note that, to leading order, the $p$-equation (3.6) decouples from the other equations and around a black hole 

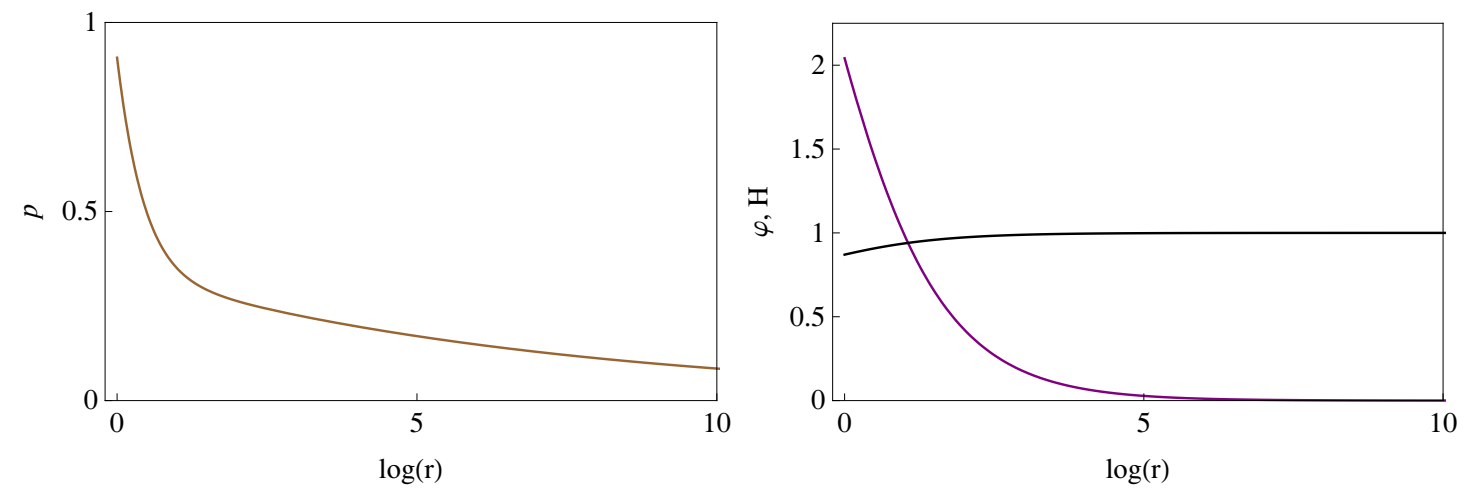

Figure 3. The left plot shows a perturbation of $p$ for $\hat{m}=\sqrt{10}-2$ whose asymptotic behaviour is consistent with (3.9). The right plot shows perturbations of $\varphi$ (in purple) and $H$ (in black) for $\hat{m}=3 / 2$, which are consistent with (3.9).

background is

$$
\left[\left(1-\frac{r_{+}^{3}}{r^{3}}\right)\left(r^{2} p\right)^{\prime}\right]^{\prime}=\left(\hat{m}^{2}+4 \hat{q}^{2}\right) p=\left(\frac{36 \hat{m}^{2}-36-\hat{m}^{4}}{4 \hat{m}^{2}}\right) p .
$$

Writing $x=\left(r_{+} / r\right)^{3}$ and definining

$$
\nu_{ \pm}=\left( \pm \sqrt{\left(36-\hat{m}^{2}\right)\left(\hat{m}^{2}-1\right)}-\hat{m}\right) / 6 \hat{m}
$$

we obtain a closed solution for $P=r^{2} p$ in terms of hypergeometric functions:

$$
\begin{aligned}
P(r)= & \Gamma\left[2 \nu_{-}+4 / 3\right] \Gamma\left[\nu_{+}+4 / 3\right] \Gamma\left[\nu_{+}\right] x^{\nu_{+}}{ }_{2} F_{1}\left[\nu_{+}, \nu_{+}+4 / 3,2 \nu_{+}+4 / 3 ; x(r)\right] \\
& -\Gamma\left[2 \nu_{+}+4 / 3\right] \Gamma\left[\nu_{-}+4 / 3\right] \Gamma\left[\nu_{-}\right] x^{\nu_{-}}{ }_{2} F_{1}\left[\nu_{-}, \nu_{-}+4 / 3,2 \nu_{-}+4 / 3 ; x(r)\right],
\end{aligned}
$$

where the constants are chosen to give a nonsingular combination at $x=1$, the position of the horizon. A quick glance at the eigenvalue plot, figure 2 , shows that, unless $\hat{m}<\sqrt{10}-2$, the $\nu_{+}$hypergeometric function will blow up at infinity. Thus for nonsingular linearised solutions we take $\hat{m}<\sqrt{10}-2$ (this does not mean that charged black holes do not exist for $\hat{m}>\sqrt{10}-2$, simply that they have strong gravitational backreaction). Figure 3 (on the left) shows a representative sample charged $B$-field around an adS black hole with $\hat{g}^{2}=52 / 5$. We will further numerically explore these black holes in section 4 .

\subsubsection{Scalar charge}

From the perspective of the $4 \mathrm{D}$ geometry, the breather mode of the hyperbolic geometry appears as a 4D scalar, and indeed the scalar equations, (3.2), (3.3) are independent of perturbations of the geometry at linear order. Extracting these scalar equations gives a second order system:

$$
\mathcal{L}\left(\begin{array}{c}
\delta \varphi \\
\delta H
\end{array}\right)=\left[\begin{array}{cc}
\left(3 \hat{m}^{2}-\hat{g}^{2}\right) / 2 & 2 \hat{q}^{2} \\
2 \hat{q}^{2} & 2\left(\hat{q}^{2}+3\right)
\end{array}\right]\left(\begin{array}{c}
\delta \varphi \\
\delta H
\end{array}\right)
$$


where $\mathcal{L}$ is the linear operator

$$
\mathcal{L} X=\frac{1}{r^{2}} \frac{d}{d r}\left[r^{4}\left(1-\frac{r_{+}^{3}}{r^{3}}\right) \frac{d X}{d r}\right] .
$$

Diagonalising the matrix on the r.h.s. of (3.14) yields two eigenvalues and eigenvectors which correspond to the two pairs of exponents indicated by the grey dashed and black dotted lines in figure 2. The fully coupled system also has perturbations of the geometry, although the asymptotic exponents indicated in figure 2 represent the fall-off at large $r$. The eigenvalues of (3.14), however, are linearized solutions around the given black hole background for all $r$. Clearly, examining the large $r$ behaviour from figure 2 shows that the dotted grey branch cannot yield a solution which is regular at both horizon and infinity: hence these branches have significant backreaction on the geometry. However, for $\hat{m}<(6-\sqrt{6}) / \sqrt{5}$ the black dotted branch gives the regular solution:

$$
\left(\begin{array}{c}
\delta \varphi \\
\delta H
\end{array}\right)=\left(\begin{array}{c}
-8 \hat{m}^{2} \pm \sqrt{36-60 \hat{m}^{2}+89 \hat{m}^{4}} \\
5 \hat{m}^{2}-6
\end{array}\right) X\left[\left(\frac{r_{+}}{r}\right)^{3}\right]
$$

where

$$
X[x]=\Gamma\left[2 \mu_{-}\right] \Gamma\left[\mu_{+}\right]^{2} x^{\mu_{+}}{ }_{2} F_{1}\left[\mu_{+}, \mu_{+}, 2 \mu_{+} ; x\right]-\Gamma\left[2 \mu_{+}\right] \Gamma\left[\mu_{-}\right]^{2} x^{\mu_{-}}{ }_{2} F_{1}\left[\mu_{-}, \mu_{-}, 2 \mu_{-} ; x\right]
$$

and

$$
\mu_{ \pm}=\frac{1}{2}[1 \pm \sqrt{1+4 \lambda / 9}]
$$

are given in terms of the eigenvalue

$$
\lambda=\frac{1}{8 \hat{m}^{2}}\left(3 \hat{m}^{4}+36 \hat{m}^{2}-36+\left(\hat{m}^{2}-6\right) \sqrt{36-60 \hat{m}^{2}+89 \hat{m}^{4}}\right) .
$$

As is noted in [47] the straight part of the dotted black curve, where $\sqrt{6 / 5}<\hat{m}<$ 1.254 , indicates an imaginary exponent. This occurs when $\lambda<-9 / 4$ in (3.19) and is analogous to a mass violating the Breitenlohner-Freedman bound, [48, 49]. This fact is indicative of a possible instability, most likely a flow from one adS branch to another. Figure 3 (on the right) shows the field profiles for the representative value $\hat{m}=3 / 2$.

\subsection{Lifshitz solutions}

We now apply to Lifshitz configurations the same techniques we developed to characterize adS solutions. As we will see, the intuition we developed for adS will help in characterizing the richer Lifshitz configurations. Unlike the adS case, there is no straightforward analytic Lifshitz black hole solution for our system. ${ }^{3}$ That this will be the case can be seen by analyzing the Lifshitz fixed point, where all of the eigenvectors corresponding to perturbations around the asymptotic Lifshitz geometry generically have components in every field. In this instance, there is no pure geometric deformation to the Lifshitz space, and any deformation necessarily includes a scalar and gauge profile.

\footnotetext{
${ }^{3}$ See however appendix B for examples of exact analytic solutions in similar dilatonic theories.
} 
The analysis of the Lifshitz point is given in appendix A, and the system of eigenvalues plotted in figure 4. Comparing this plot to the adS case, we see that the eigenvalues are symmetric around $-(z+2) / 2$, and in particular, an eigenvalue $\Delta=-(z+2)$ exists, which is continuous with the pure black hole adS eigenvalue, $\Delta=-3$. Indeed, plotting the Lifshitz and adS eigenvalues side by side shows how the perturbations around the critical points merge as $z \rightarrow 1$ or $\hat{m} \rightarrow \sqrt{10}-2$. Using the intuition obtained from analysing the adS solutions, one might expect that the mass and charge perturbations continue from the adS side into the Lifshitz side. However, things are not so straightforward, and are actually more interesting. While the subspace spanned by the two eigenvectors clearly is the same on each solution branch, the eigenvector basis need not be: the adS eigenvectors are either pure charge or pure geometry, whereas the Lifshitz perturbations include all fields. A careful tracking of the perturbations as $z \rightarrow 1$ indicates that the Lifshitz perturbations can be thought as corresponding to a $\pi / 4$ rotation of the adS perturbations. Thus, the " $-(z+2)$ " eigenvector is actually a charged black hole, most likely an extremal black hole given the combination of adS eigenvectors.

It is worth exploring in more detail the analytic expansions of the functions, especially for the special cases identified above. First of all, by analysing the linear perturbations for the eigenvalue $\Delta=-(z+2)$, we find the solution (for general $z$ ):

$$
\begin{aligned}
\delta \varphi= & \frac{2 M \sqrt{z-1}}{r^{z+2}}\left[\sqrt{2(4+z)}\left(-48-8 z+14 z^{2}+4 z^{3}\right)\right. \\
& \left.+\left(136+40 z-40 z^{2}-21 z^{3}-2 z^{4}\right)\right] \\
F= & 1-\frac{2 M \sqrt{z-1}}{(2+z) r^{z+2}}\left[\sqrt{2(4+z)}\left(96-352 z-228 z^{2}-22 z^{3}+4 z^{4}\right)\right. \\
& \left.+\left(-272+952 z+760 z^{2}+146 z^{3}-11 z^{4}-11 z^{5}-2 z^{6}\right)\right] \\
D= & 1+\frac{2 M \sqrt{z-1}}{r^{z+2}}\left[\sqrt{2(4+z)}\left(-48+40 z+38 z^{2}+4 z^{3}\right)\right. \\
& \left.+\left(136-96 z-124 z^{2}-19 z^{3}+7 z^{4}+2 z^{5}\right)\right] \\
H= & +\frac{2 M \sqrt{z-1}}{r^{z+2}}\left[\sqrt{2(4+z)}\left(-48+8 z+2 z^{2}\right)+\left(136-8 z-12 z^{2}+5 z^{3}+2 z^{4}\right)\right] \\
p= & \sqrt{z-1}+\frac{2 M}{r^{z+2}}\left[\sqrt{2(4+z)}\left(-48-8 z+22 z^{2}+6 z^{3}\right)\right. \\
& \left.+\left(136+40 z-64 z^{2}-23 z^{3}+7 z^{4}+2 z^{5}\right)\right]
\end{aligned}
$$

where $M$ is some integration parameter. This solution is valid at first order in perturbations around the pure Lifshitz solution with arbitrary dynamical exponent $z$, and makes manifest that all fields are normally switched on for Lifshitz configurations. We additionally checked that the system of equations can be solved also at next to leading order, providing corrections to the above profiles that scale as $M^{2} / r^{2(z+2)}$ : the expression for the $z$-dependent coefficients is however too long to be presented here. Notice that the limit $z \rightarrow 1$ is well behaved, and leads apparently to the perturbative pure charge adS solution we discussed in equation (3.10). 

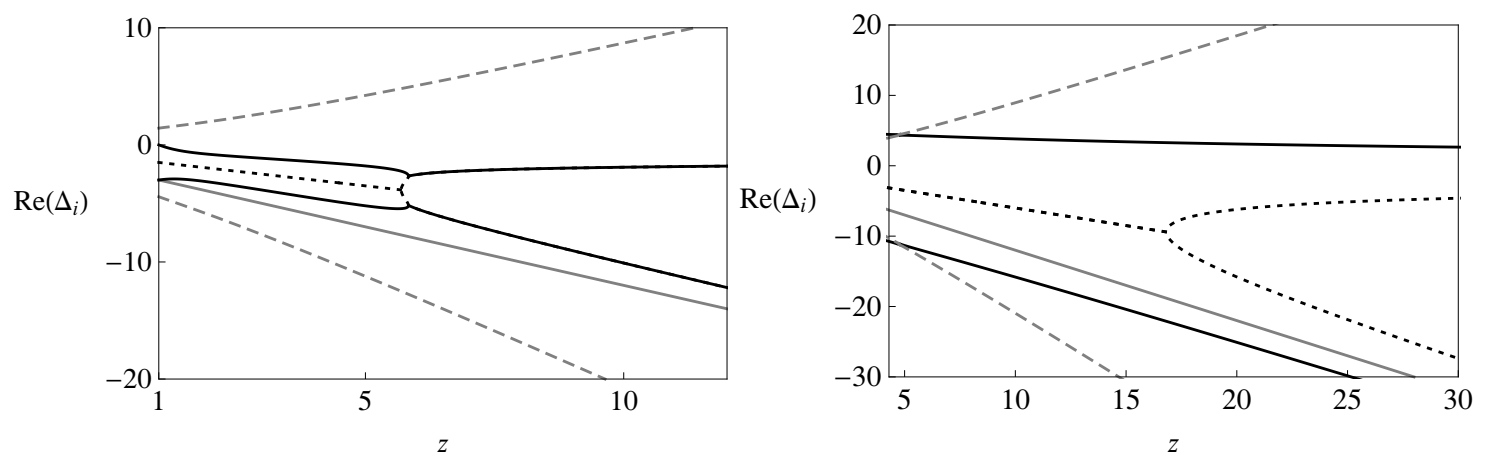

Figure 4. Plots of the real parts of the eigenvalues of perturbations around the Lifshitz solution as a function of $z$. The left and right plots correspond to the upper and lower sign choices in (2.11) respectively. For the upper sign choice, the eigenvalues are all real only in the region $5.69<z<5.83$, whereas for the lower sign choice one finds real eigenvalues for $z>16.82$.

We can also characterize the solution corresponding to the black line in the left panel of figure 4 , that joins with the grey line when $z \rightarrow 1$. In order to do this, we expand the eigenvalues tending to the $\Delta=-3$ near $z \rightarrow 1$ to obtain:

$$
\begin{aligned}
& \Delta_{1}=-3-(z-1) \\
& \Delta_{2}=-3+\frac{1}{189}(260 \sqrt{10}-701)(z-1)+\mathcal{O}\left[(z-1)^{2}\right]
\end{aligned}
$$

The first is just the eigenvalue $\Delta=-z-2$ rewritten as an expansion around $z=1$, with a corresponding solution, (3.20), that can also be expanded near $z=1$. The second eigenvalue, $\Delta_{2}$, is instead that of the black curve in figure 4 , and we can similarly determine its corresponding solution. To leading order in $z-1$ we find:

$$
\begin{aligned}
\delta \varphi_{1} & =\frac{\mu \sqrt{z-1}}{126 r^{\Delta_{1}}}(31-40 \sqrt{10}), & \delta \varphi_{2} & =\frac{\mu \sqrt{z-1}}{126 r^{\Delta_{2}}}(31-40 \sqrt{10}) \\
F_{1} & =1-\frac{\mu \sqrt{z-1}}{63 r^{\Delta_{1}}}(65 \sqrt{10}-149), & F_{2} & =1-\frac{\mu \sqrt{z-1}}{3 r^{\Delta_{2}}} \\
D_{1} & =1+\frac{\mu \sqrt{z-1}}{63 r^{\Delta_{1}}}(11+25 \sqrt{10}), & D_{2} & =1+\frac{\mu \sqrt{z-1}}{63 r^{\Delta_{2}}}(139-40 \sqrt{10}) \\
H_{1} & =1+\frac{\mu \sqrt{z-1}}{126 r^{\Delta_{1}}}(101-20 \sqrt{10}), & H_{2} & =1+\frac{\mu \sqrt{z-1}}{126 r^{\Delta_{2}}}(101-20 \sqrt{10}) \\
p_{1} & =\sqrt{z-1}+\frac{\mu}{r^{\Delta_{1}}}, & p_{2} & =\sqrt{z-1}+\frac{\mu}{r^{\Delta_{2}}}
\end{aligned}
$$

for some integration constant $\mu$. Notice that both these eigenvector solutions approach the pure charge adS solution for in the limit $z \rightarrow 1$. On the other hand, for small values of $(z-1)$ they differ (by an identical amount) only in the metric components $F$ and $D$ (up to corrections suppressed by powers of $\sqrt{z-1}$ ). The difference in the eigenvectors is consequently due to contributions of pure geometry to the Lifshitz configuration, that we know corresponds to the adS-Schwarzschild eigenvector in the pure adS case. In this sense, we can regard the two eigenvectors (3.23), in the limit of small $(z-1)$, as if each forming a $\pi / 4$ degree angle with the eigenvectors of asymptotically adS configurations. 


\section{Numerical black hole solutions}

Having developed an analytical understanding of the asymptotic properties of the solutions of the system, we now present some numerical solutions to the fully coupled system of field equations. In this section, we begin by computing and analysing adS black hole solutions, followed by their Lifshitz generalizations, and then uplift the solutions to type IIA supergravity.

To obtain black hole solutions for our system we must ensure that our boundary conditions are consistent with the nature of the near horizon region of a black hole spacetime. These conditions will be the same irrespective of whether we are interested in asymptotically Lifshitz or adS black holes. Assuming that the horizon is non-degenerate, we wish the $g_{t t}$ component of the metric to have a simple zero and the $g_{r r}$ to have a simple pole at $r=r_{+}$. Checking that the matter and metric fields and the energy momentum tensor are regular at the horizon imposes no further constraints and we find the near horizon expansion of the fields to be

$$
\begin{aligned}
F & =f_{1}\left(r-r_{+}\right)+f_{2}\left(r-r_{+}\right)^{2}+\ldots \\
D & =d_{1}\left(r-r_{+}\right)+d_{2}\left(r-r_{+}\right)^{2}+\ldots \\
H(r) & =H_{0}+H_{1}\left(r-r_{+}\right)+H_{2}\left(r-r_{+}\right)^{2}+\ldots \\
\varphi(r) & =\varphi_{0}+\varphi_{1}\left(r-r_{+}\right)+\varphi_{2}\left(r-r_{+}\right)^{2}+\ldots \\
p(r) & =p_{0}+p_{1}\left(r-r_{+}\right)+p_{2}\left(r-r_{+}\right)^{2}+\ldots
\end{aligned}
$$

where $r_{+}$is the Schwarzschild radius of the black hole. By inserting these into the field equations and expanding order by order, appropriate boundary conditions can be found. This procedure leaves us apparently with four independent field variables at the horizon: $f_{1}, H_{0}, p_{0}$ and $\varphi_{0}$ for each choice of $z$ or $\hat{m}$. However, note that $f$ can be shifted by a constant at the price of rescaling $t$, thus $f_{1}$ is essentially a gauge degree of freedom, which is tuned to achieve $F \rightarrow 1$ at infinity. Note also that the metric and field equations are invariant under the rescaling

$$
r \rightarrow \lambda r \quad, \quad t \rightarrow \frac{t}{\lambda^{z}}, \quad x^{i} \rightarrow \frac{x^{i}}{\lambda}
$$

which means that we are free to set the Schwarzschild radius of the black hole, $r_{+}$, to 1 . We choose, however, to keep $r_{+}$explicit in our expressions for clarity.

Numerical solutions can be found by fixing either $\hat{m}$ (for adS) or $z$ (for Lifshitz) and using a shooting method to integrate out from the horizon, tuning the inital data to give a regular asymptotic solution.

\subsection{AdS black holes}

As a warm up, consider first the asymptotically adS solutions. For numerical simplicity we focus on the case where there is only one unphysical growing mode at infinity, i.e. $\hat{m} \in[\sqrt{6 / 5}, \sqrt{10}-2]$. Note that in this range the exponents of $\varphi$ and $H$ are complex. Via this process we find a two parameter family of asymptotically adS black hole solutions 

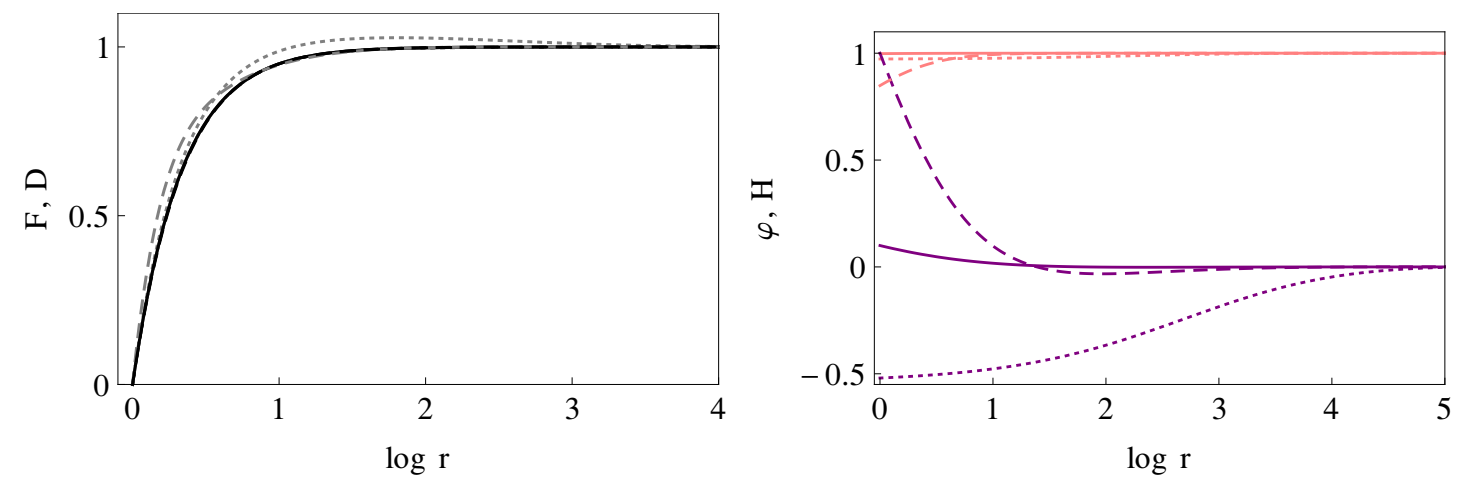

Figure 5. Field profiles for asymptotically adS black holes with $\hat{m}=1.105$ and no $B$-charge. In the left figure, the black lines correspond to the $F$ function and the grey to $D$. On the right plot, the purple lines correspond to the dilaton, and the pink to the $H$ field. In both plots the solid lines are with a small perturbation, $\varphi_{0}=0.2$, the dashed and dotted plots to a larger dilaton charge, with $\varphi_{0}=1$ and $\varphi_{0}=-0.52$ respectively.

for a fixed value of $\hat{m}$. In light of the previous analytic findings these correspond to some combination of gauge and scalar charge. A priori we are free to choose any two of $H_{0}, \varphi_{0}$ and $p_{0}$ as our free parameters and we shall choose them to be $\varphi_{0}$ and $p_{0}$. Figures 5 to 7 show examples of the asymptotically adS black hole solutions with $\hat{m}=1.105$.

Figure 5 shows three solutions where $p \equiv 0$, in which the black holes have only scalar charge. One has only a small scalar at the horizon, and the other two a more substantial dilaton charge, one positive and one negative. The small dilaton perturbation leads to a smaller perturbation in $H$ and largely leaves $F$ and $D$ unchanged, which is consistent with the approximations made in finding the analytic solution (3.17). Turning $\varphi_{0}$ up to 1 shows how positive dilaton charge reacts on the geometry. The perturbation in $H$ grows and $F$ and $D$ are no longer equal, however, all three functions remain monotonically increasing outside the horizon. Something more interesting happens however if we try to lower the value of the scalar at the horizon, i.e. letting $\varphi_{0}<0$. Overall, very little happens to the geometry, however, as can be seen from the plot for $\varphi_{0}=-0.52$, the $D$ and $H$ functions cease to be monotonic and all the fields relax to their asymptotic values significantly more slowly than for positive scalar charge. Indeed, there is a critical value of $\varphi_{0} \simeq-0.53$, below which the charged black hole solution ceases to exist. This is because there is a runaway behaviour in $\hat{V}(\varphi)$ for $\varphi$ too negative. This critical value shifts towards the origin as we turn on $B$-charge, as can be seen by looking at the source for the $\varphi$ equation of motion at the horizon.

Figure 6 shows solutions with $B$-charge, while keeping $\varphi_{0}=0$. Again, we show the comparison between a small and larger gauge charge. The small perturbation in $P$ leaves the other fields largely unchanged, and comparing the analytic approximate and numerically generated profiles of $P$ for the same value of $\hat{m}$, one finds that the two appear identical to the naked eye. Increasing $p_{0}$ alters all the other fields as seen in the dashed plots, and once again $F$ and $D$ increasingly differ.

The effect of both scalar and gauge charge on the black hole is shown in figure 7 . 

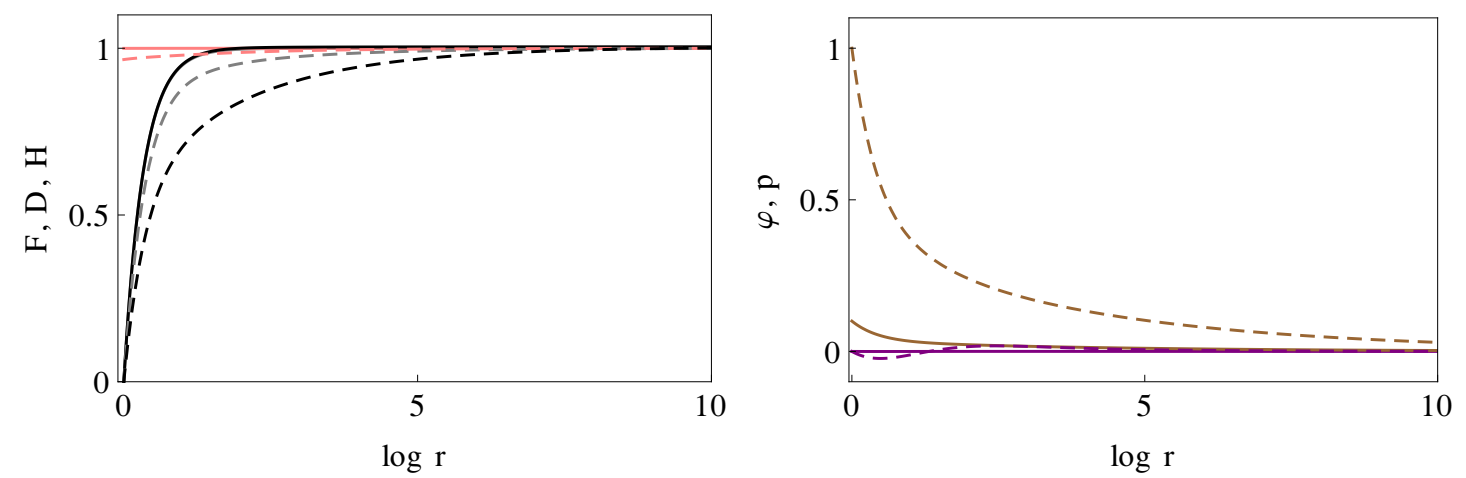

Figure 6. Field profiles for asymptotically adS black holes with $\hat{m}=1.105$, with $\varphi_{0}=0$. As before, the black, grey, pink and purple lines correspond to the $F, D, H$ and $\varphi$ fields, with the $p$-field being plotted in brown. The solid lines are for a small perturbation, $p_{0}=0.1$, and the dashed to a larger charge, $p_{0}=1$.
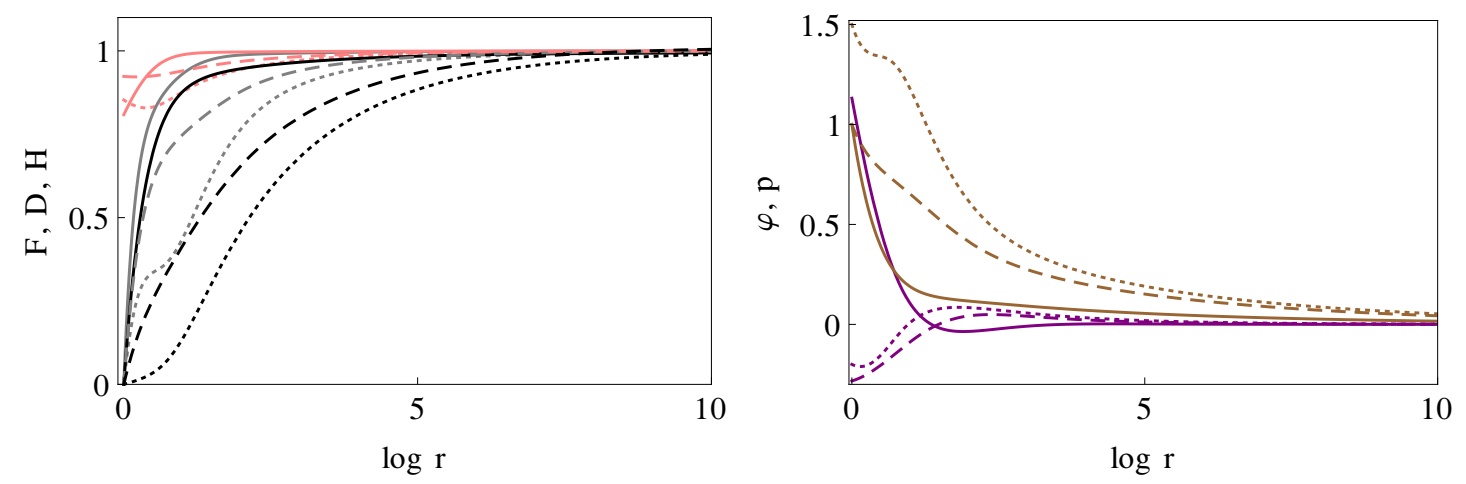

Figure 7. Field profiles for generic adS black holes with $\hat{m}=1.105$. The solid lines correspond to horizon data $\varphi_{0}=4 \sqrt{2} / 5, p_{0}=1$; the dashed lines to $\varphi_{0}=-\sqrt{2} / 5, p_{0}=1$; and the dotted lines to $\varphi_{0}=-0.2, p_{0}=1.5$. The colour coding of the plot is the same as figure 6 .

Here, the dotted line shows a nearly critical negative scalar charge black hole, in which the $B$-charge has been turned to near extremality (the temperature of this black hole is $\left.0.024 r_{+}\right)$.

\subsection{Lifshitz black holes}

We now turn to black hole solutions that asymptote the Lifshitz spacetime defined by (2.11). A crucial difference between this case and the asymptotically adS case is that the background 2 -form gauge field is now nonzero, $p \neq 0$. As a result we were unable to find an exact analytic expression for the black hole similar to (3.9), only perturbative or asymptotic solutions. We must therefore rely solely on numerical results for full solutions to the equations of motion. Another difference with the adS case is that all of the fields necessarily participate in the black hole solution. That this is the case can be seen by checking the eigenvectors of the perturbations around the critical point. All of the eigenvectors have (different) combinations of scalar, gauge and geometry components. Thus we expect that, for Lifshitz black holes, nontrivial profiles of all of the fields will generally be present. 

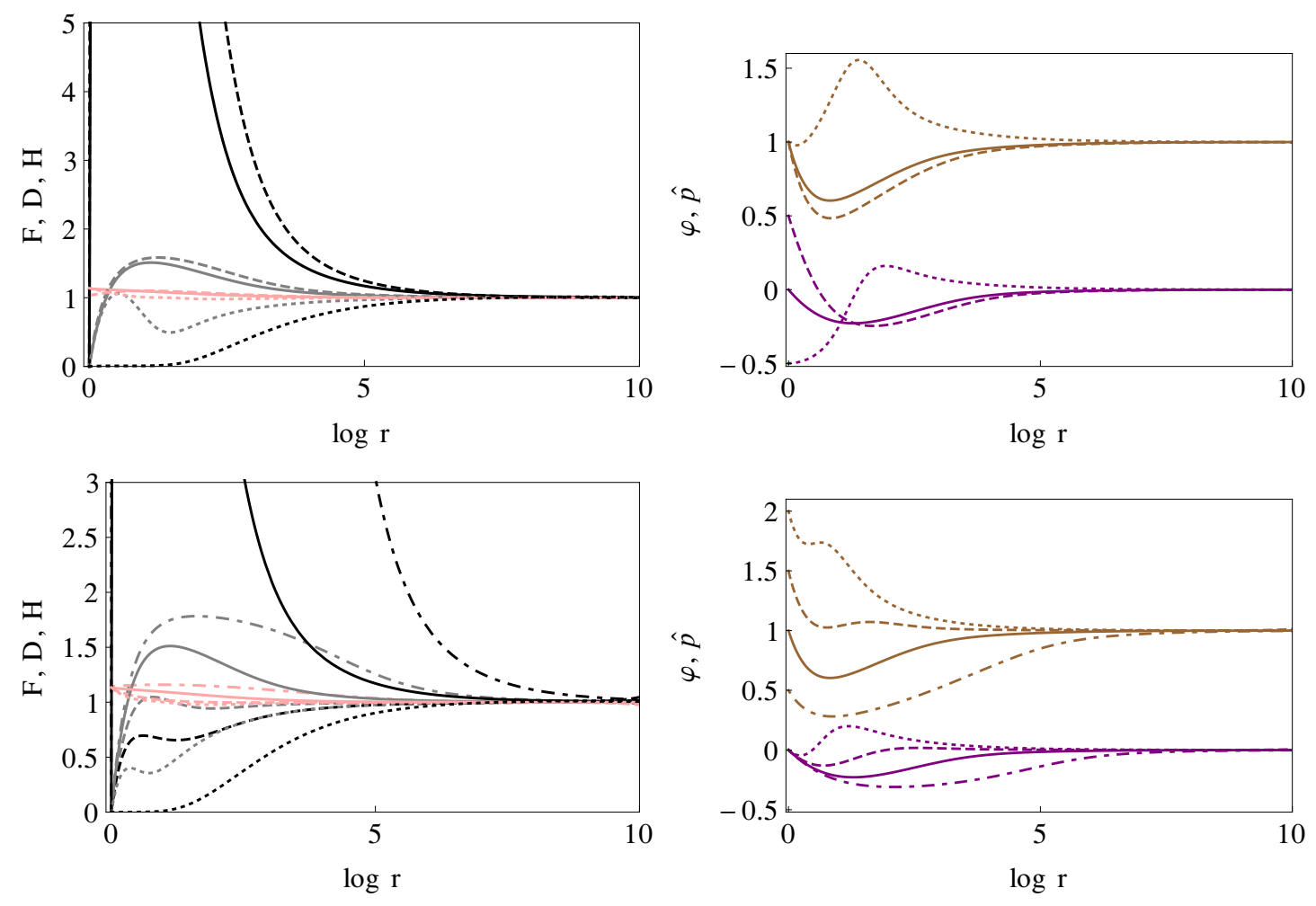

Figure 8. A selection of plots showing the field profiles for asymptotically Lifshitz black holes for $z=2$. The colours are the same as for ads, with $F, D, H, \varphi$ and $\hat{p}=p / \hat{b}$ being displayed in black, grey, pink, purple and brown respectively. The upper pair of plots explore the effect of varying $\varphi_{0}$, and the lower plots the impact of changing $\hat{p}_{0}$. In each case, the labelling of the curves is defined by the initial conditions in the right hand plot.

The numerical solutions are found in precisely the same way as in the adS case with the only difference being that the parameters of the theory are now defined by (2.11) where the dynamical exponent $z \geq 1$ is used to fix the theory as opposed to $\hat{m}$. For simplicity we present solutions corresponding to the upper sign choice in (2.11) as this joins the adS branch and is more stable to integrate. By integrating the equations of motion (3.2) to (3.6) we once again find a two parameter family of asymptotically Lifshitz black hole solutions for each value of $z$ to which we assign the free parameters $\varphi_{0}$ and $p_{0}$. Using intuition from the adS case we suggest that these parameters relate to the scalar and $B$-charge of the black hole, however, since all the fields participate in any asymptotic fall-off to the Lifshitz spacetime, this relation will not be completely straightfoward.

In all of the Lifshitz plots, we renormalize the $z$-dependence of the gauge field by plotting $\hat{p}=p / \hat{b}$, so that unnecessary variation with $z$ is scaled out. Figure 8 explores the impact of varying the gauge and scalar initial conditions on a $z=2$ Lifshitz black hole. The plots are reasonably self-explanatory, exploring the impact of altering $\varphi_{0}$ (upper) and $\hat{p}_{0}$ (lower) relative to the fiducial black hole solution shown in each case by solid lines, whose horizon values of the dilaton and $B$-field are the same as the asymptotic values. Most of these black hole solutions (see also figure 9) have extremely strongly warped geometries 

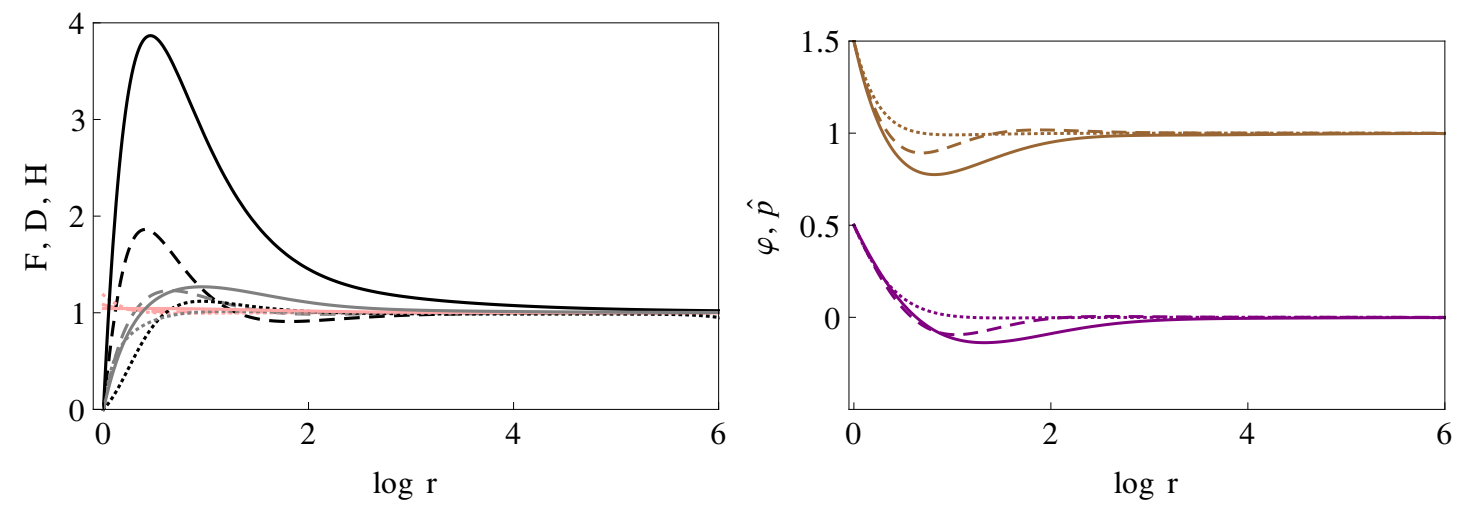

Figure 9. Plots showing the field profiles for asymptotically Lifshitz black holes with $\varphi_{0}=0.5$, $\hat{p}_{0}=1.5$ at different $z$. The black, grey, pink, blue and red lines correspond to $F, D, H, \hat{p}$ and $\varphi$ respectively. The solid lines correspond to $z=2$, the dashed lines to $z=3$, and the dotted lines to $z=5.75$.

near the horizon, with the "Newtonian potential", $F$, rising very sharply to a rather high maximum before falling to its asymptotic value of 1 from above. For example, in the fiducial solution, the maximum of $F$ is around 30 (in units of $r_{+}$), and lowering the horizon value of $\hat{p}_{0}$ only exacerbates this effect.

In the upper plots, showing some sample solutions for different $\varphi_{0}$, we see that decreasing $\varphi_{0}$ below zero damps the $F$ potential, which now looks more like a canonical black hole, with the example shown in the dotted plot, $\varphi_{0}=-0.5$ being representative of a nearly 'extremal' black hole, in the sense that the solution will cease to exist if $\varphi$ is lowered further (for the same reason as in the adS case) and also in the sense of the temperature dropping to zero. Increasing $\varphi_{0}$ on the other hand has the opposite effect, with $\varphi_{0}=0.5$ increases the sharp peak of $F$, which now has a maximum of around 80 (the dashed lines). Interestingly however, this variation with the dilaton horizon value is not monotonic, and as $\varphi_{0}$ is increased further, the amount of warping peaks, then subsides, and as we will see in the next section when we consider thermodynamics, this behaviour is mirrored in the temperature of the black hole dropping to zero.

In the lower pair of plots, which explore changing the horizon value of the gauge field, we see that increasing $\hat{p}_{0}$ rapidly restores the $F$ field to a more canonical form, in the case of the dashed $\left(\hat{p}_{0}=1.5\right)$ and dotted $\left(\hat{p}_{0}=2\right)$ plots. Since the temperature of these black holes drops, increasing the horizon value of $\hat{p}$ can be seen to be analogous to charging up a Reissner-Nordstrom black hole. Correspondingly, as can be seen in the dot-dashed plot, dropping $\hat{p}_{0}$ below its asymptotic value causes the black hole to become more strongly warped, and as we will see, hotter.

Altering both $\varphi_{0}$ and $\hat{p}_{0}$ produces a combination of these effects: increasing $\varphi_{0}$ first moves the black hole away from, then towards, 'extremality', and decreasing $\hat{p}_{0}$ always moves the black hole away from extremality. Correspondingly, the maximal value of $B$-charge (tracked by $\hat{p}_{0}$ ) first increases, then decreases, as we increase the dilaton charge $\varphi_{0}$. 
Finally, the plots in figure 9 show the effect of altering the dynamical exponent, $z$, for a system with $\varphi_{0}=0.5$ and $\hat{p}_{0}=1.5$. The plots show the field profiles for $z=2$, $z=3$ and $z=5.75$, where the last value was chosen to be within the range for which all the eigenvalues of figure 4 are real. These plots show how $z$ can alter the field profiles, particularly $F$ and $D$, and that increasing $z$ hastens the convergence to the Lifshitz solution. This was to be expected since, as can be seen in figure 4, increasing $z$ largely reduces the eigenvalues governing each fields approach to the Lifshitz fixed point.

\subsection{Uplifting to type IIA in 10 dimensions}

With the $6 \mathrm{D}$ solutions in hand, it is straightforward to uplift them to configurations in Type IIA massive supergravity. Following [17, 20], we define

$$
\begin{aligned}
X(r) & =e^{\phi_{0} / \sqrt{2}}\left(\frac{g}{3 \mathrm{~m}}\right)^{1 / 4} e^{\varphi(r) / 2} \\
\mathrm{C}(\rho) & =\cos \rho, \quad \mathrm{S}(\rho)=\sin \rho \\
\Delta(\rho) & =X \mathrm{C}^{2}+X^{-3} \mathrm{~S}^{2} \\
\mathrm{U}(\rho) & =X^{-6} \mathrm{~S}^{2}-3 X^{2} \mathrm{C}^{2}+4 X^{-2} \mathrm{C}^{2}-6 X^{-2},
\end{aligned}
$$

as well as the constant $k=\left(3 \mathrm{~m} g^{3}\right)^{1 / 4} / 2$. We can then write the ten dimensional, uplifted configurations as:

$$
\begin{aligned}
d s_{10}^{2}= & \mathrm{S}^{1 / 12} X^{1 / 8}\left[\Delta^{3 / 8}\left(\mathrm{LiBH}_{4} \times \Omega_{2}\right)-2 k^{-2} \Delta^{3 / 8} X^{2} d \rho^{2}-\frac{1}{2} k^{-2} \Delta^{-5 / 8} X^{-1} \mathrm{C}^{2} \sum_{i}^{3}\left(h^{(i)}\right)^{2}\right], \\
\mathbf{F}_{4}= & \frac{\sqrt{2}}{6} k^{-3} \mathrm{~S}^{1 / 3} \mathrm{C}^{3} \Delta^{-2} U d \rho \wedge \epsilon_{3}+\sqrt{2} k^{-1} \mathrm{~S}^{1 / 3} \mathrm{C} X^{4} \star_{6} G_{3} \wedge d \rho \\
& -\frac{1}{\sqrt{2}} k^{-2} \mathrm{~S}^{1 / 3} \mathrm{C} F_{2}^{(3)} \wedge h^{(3)} \wedge d \rho+\frac{1}{4 \sqrt{2}} k^{-2} \mathrm{~S}^{4 / 3} \mathrm{C}^{2} \Delta^{-1} X^{-3} F_{2}^{(3)} \wedge \sigma^{(1)} \wedge \sigma^{(2)} \\
& +\sqrt{2} k^{-3} \mathrm{~S}^{4 / 3} \mathrm{C}^{4} \Delta^{-2} X^{-3} d X \wedge \epsilon_{3}, \\
\mathbf{G}_{3}= & 2 \sqrt{2} \frac{k^{2}}{g} \mathrm{~S}^{2 / 3} G_{3}, \quad \mathbf{F}_{2}=0 \\
e^{\Phi}= & \mathrm{S}^{-5 / 6} \Delta^{1 / 4} X^{-5 / 4}
\end{aligned}
$$

where

$$
h^{(i)}=\sigma^{(i)}-g A_{1}^{(i)},
$$

with $\sigma^{(i)}$ the left-invariant 1-forms on $S^{3}$, and $\epsilon_{3}=h^{(1)} \wedge h^{(2)} \wedge h^{(3)}$. The parameters of the $6 \mathrm{D}$ theory are related to the Type IIA mass parameter via $\mathbf{m}=\left(2 \mathrm{~m} g^{3} / 27\right)^{1 / 4}$. The uplift gives us some insight into the kinds of sources in 10D that give rise to the adS and Lifshitz black holes in $4 \mathrm{D}$. Notice that the ten dimensional $R R \mathbf{F}_{\mathbf{2}}$ field strength vanishes, while the RR $\mathbf{F}_{\mathbf{4}}$ field and the NS $\mathbf{G}_{\mathbf{3}}$ field are switched on in several directions. The main difference between the black hole solutions discussed here and their pure Lifshitz/adS counterparts presented in [17] is in the non-trivial $r$-profiles, which imply that $\mathbf{F}_{4}$ contains an additional component in the direction $d X \wedge \epsilon_{3}$. The configurations could be interpreted as a system of D-branes and NS-branes, analogously to [17]. 

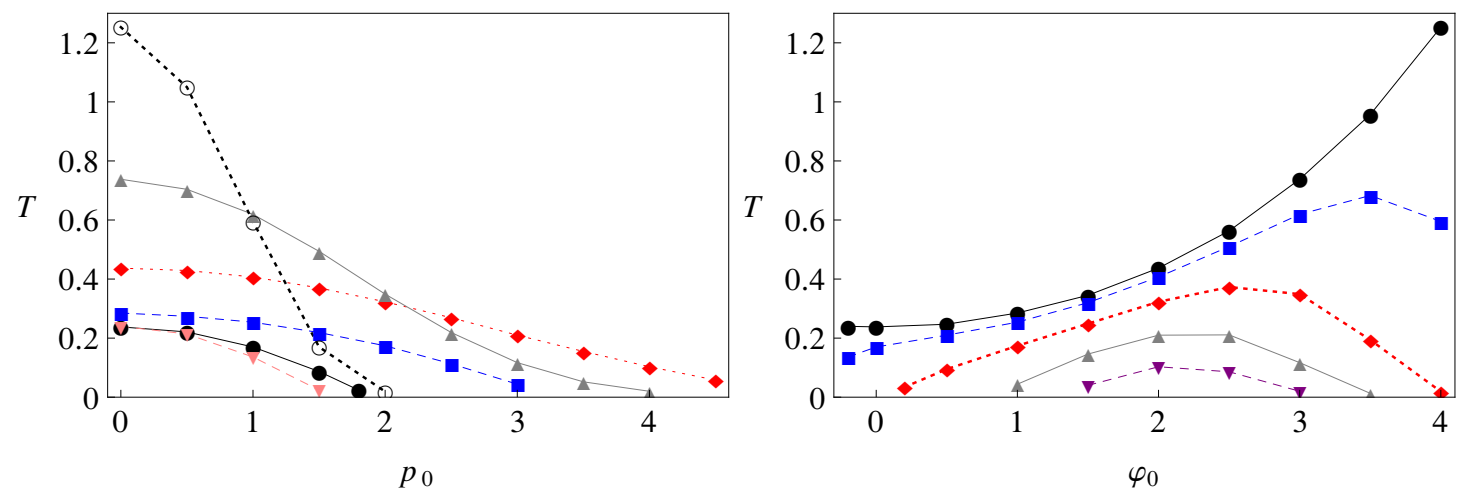

Figure 10. Plots of the temperature of an adS black hole with $\hat{m}=1.105$ shown as a function of $p_{0}$ (left) and $\varphi_{0}$ (right). In the left plot, the dashed pink data in inverted triangles corresponds to $\varphi_{0}=-0.2$; the black data in dots to $\varphi_{0}=0$; the dashed blue with squares to $\varphi_{0}=1$; the dotted red with diamonds to $\varphi_{0}=2$; the grey triangles to $\varphi_{0}=3$; and the dashed black with open circles to $\varphi_{0}=4$. In the right plot, the lines run from $p_{0}=0$ in black with dots at the top, to $p_{0}=4$ in dashed purple with inverted triangles at the bottom in increments $\Delta p_{0}=1$.

\section{Thermodynamics}

Having determined the black hole solutions for our supergravity set-up, it is interesting to investigate some of their general properties besides their field profiles. In this section we make the first few steps of this exploration by studying the dependence of the temperature of these black holes on the initial parameters $\varphi_{0}$ and $p_{0}$, as well as commenting on entropy.

The temperature of the black hole is given by

$$
T=\left.\frac{r_{+}^{z+1}}{4 \pi} \sqrt{D^{\prime} F^{\prime}}\right|_{r=r_{+}},
$$

which is calculated directly from the numerical solutions. Figures 10 and 11 show the temperatures of both asymptotically adS, and asymptotically Lifshitz black holes as a function of our initial parameter $\hat{p}_{0}$ and $\varphi_{0}$. In all the temperature plots, we have shown the temperature normalized at $r_{+}=1$ for simplicity. We discuss the $r_{+}$dependence of the temperature at the end of this section.

In figure 10, plots are shown of the temperature of an asymptotically adS black hole with $\hat{m}=1.105$. On the left, the plot is shown as a function of the $B$-charge for a range of $\varphi_{0}$, on on the right as a function of $\varphi_{0}$ for a range of $p_{0}$. The left plot shows the expected behaviour of a charged black hole, in that adding charge reduces temperature monotonically to zero at an extremal limit. The effect of scalar charge in this case is more interesting. At zero $B$-charge, the impact of increasing $\varphi_{0}$ is to increase the temperature of the black hole, and one might expect therefore that the allowed $B$-charge range is increased. However, an interesting phenomenon occurs. As the black hole becomes more and more charged under the scalar field, the maximal amount of $B$-charge we are able to add starts to drop, and at very high scalar charges we can no longer add much gauge charge. That the "extremal" limit should not be a simple sum of the two charges, but 

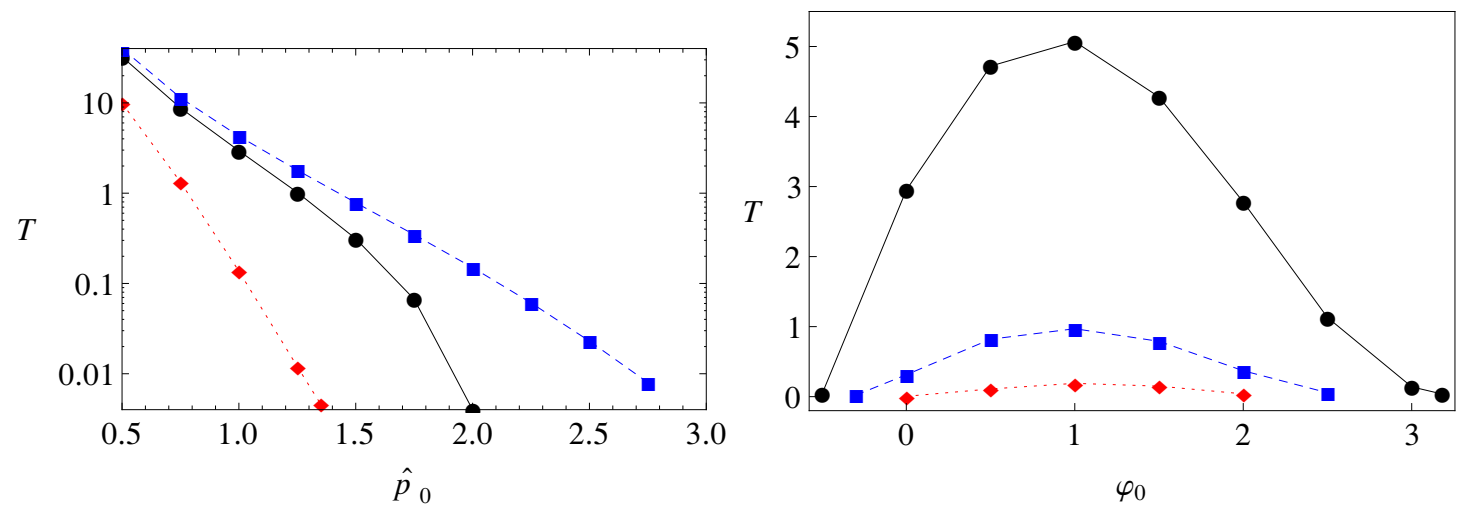

Figure 11. Plot of the temperature of asymptotically Lifshitz black holes for varying $\hat{p}_{0}$ and $\varphi_{0}$, with $z=2$. On the left the plot depicts the temperatures of $z=2$ black holes as a function of $\hat{p}_{0}$ for $\varphi_{0}=0$ in black with circular data points, $\varphi_{0}=1.5$ in dashed blue with square data points, and for $\varphi_{0}=3$ in dotted red with diamond data points. On the right, the temperature is shown as a function of $\varphi_{0}$ for $\hat{p}_{0}=1$ in black (circles), $\hat{p}_{0}=1.5$ in blue (dashed/squares), and $\hat{p}_{0}=2$ in red (dotted/diamonds).

some more complex combination is an interesting difference from most black holes with more than one charge.

Figure 11 shows the corresponding plots for a Lifshitz black hole, with $z=2$ taken as an example. As with the adS black hole, increasing $\hat{p}_{0}$ reduces the temperature, and once again, we see that there is a finite range of $\varphi_{0}$ for which the black holes exist. However, because the Lifshitz spacetime has a nonzero background $B$-field, there is a clear difference in the temperature as a function of $\hat{p}_{0} \rightarrow 0$. For the Lifshitz black hole, the temperature increases sharply as we reduce the initial value of $\hat{p}_{0}$, and would appear to diverge as $\hat{p} \rightarrow 0$. In the absence of analytic arguments we cannot say definitively that $T$ diverges, however, our numerical integrations become more and more extreme as we reduce $\hat{p}_{0}$. It is worth noting that we have presented our temperature plots renormalized to $r_{+}=1$, clearly, dropping $r_{+}$drops the temperature, so it is possible that we can achieve $\hat{p}_{0} \rightarrow 0$ by taking $r_{+} \rightarrow 0$, indeed, such a spacetime would represent a flow from a Lifshitz space in the UV to an adS space in the IR, [47]. However, the results of [47] would indicate that this will only happen for a specific value of $\varphi_{0}$, namely, the one corresponding to an adS solution. Thus, we would expect the generic $\hat{p}_{0} \rightarrow 0$ limit to be singular.

In figure 12 we see how altering $z$ affects the temperature of the black hole by looking at the variation of temperature with $\hat{p}_{0}$ for sample values of $z$, and exploring in detail the $z$-dependence for sets of representative initial data. In general, we see that increasing $z$ raises the temperature for small $\hat{p}_{0}$ but lowers it for larger $\hat{p}_{0}$, although for temperatures close to 1 , the temperature seems to first increase then decrease with $\hat{p}_{0}$.

Finally, we consider the entropy of the black holes, which can be computed from the area of the horizon as:

$$
S=\frac{1}{4} r_{+}^{2} H\left(r_{+}\right)
$$

per unit volume (and setting $G_{6}=1$ ). Figure 13 shows how the entropy of a $z=2$ Lifshitz 

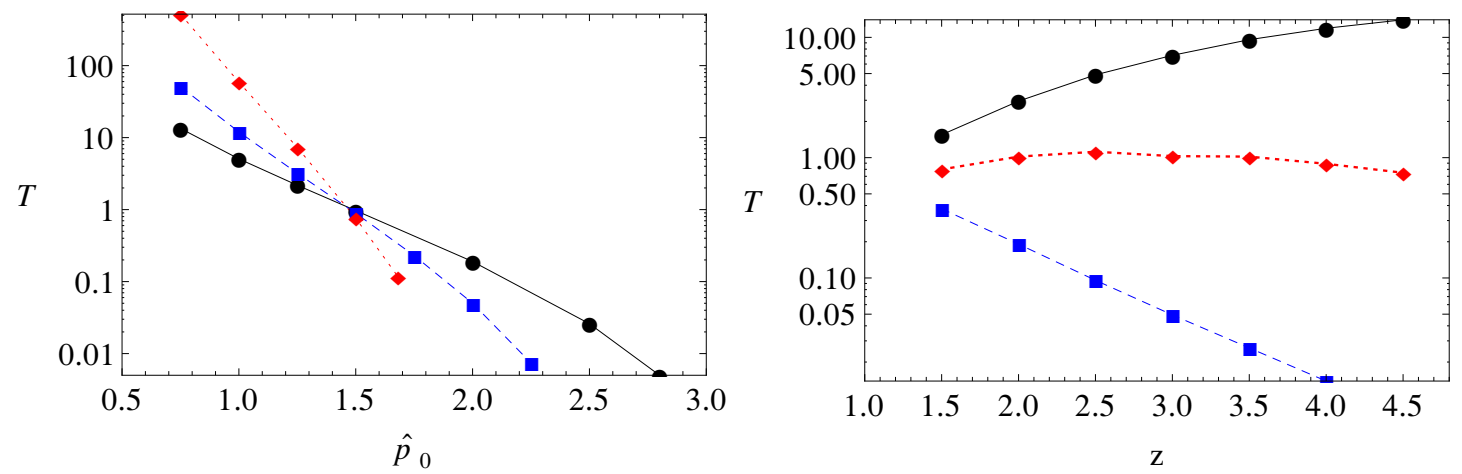

Figure 12. Plot of the temperature of asymptotically Lifshitz black holes for varying $\hat{p}_{0}, \varphi_{0}$, and $z$. On the left, the curves give the temperature of a Lifshitz black hole with $\varphi_{0}=1$ as a function of $\hat{p}_{0}$ for different values of $z$ : black (circles) to $z=2$, blue (dashed/squares) to $z=3$, and red (dotted/diamonds) to $z=5$. The three curves appear to intersect at a single point, however, the resolution of the data is insufficient to be sure if this is exact. On the right, the temperature is plotted as a function of $z$ for initial data $\left(\varphi_{0}, \hat{p}_{0}\right)=(0,1)$ in black (circles), $\left(\varphi_{0}, \hat{p}_{0}\right)=(1,2)$ in blue (dashed/squares), and $\left(\varphi_{0}, \hat{p}_{0}\right)=(0,1.25)$ in red (dotted/diamonds).
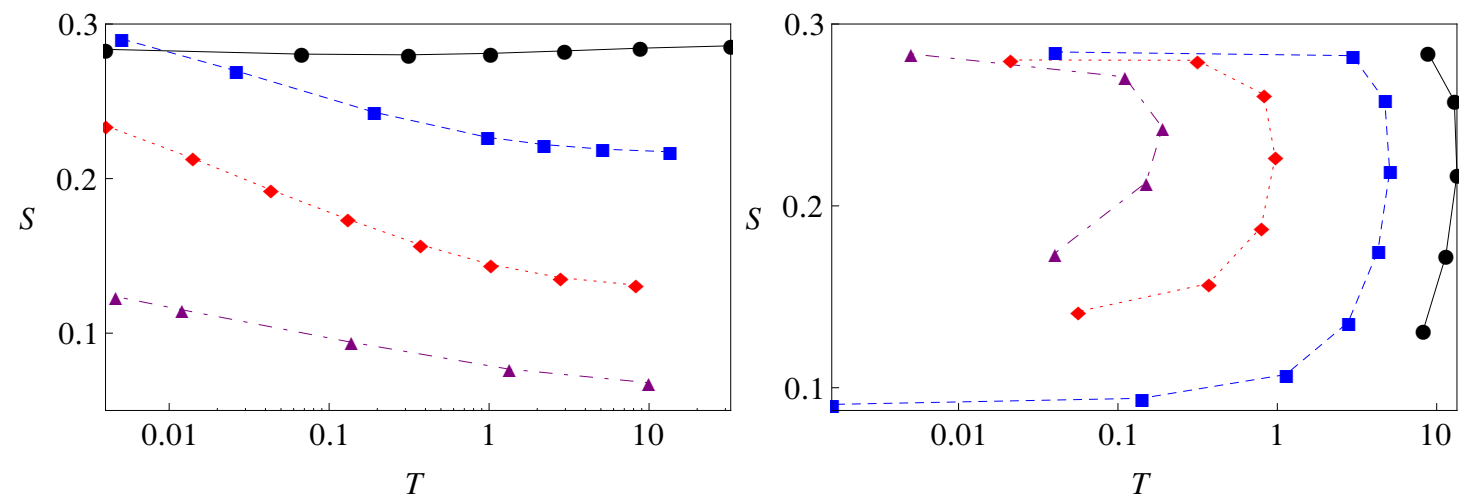

Figure 13. Plots of the entropy of a $z=2$ Lifshitz black hole as a function of the temperature with $r_{+}=1$. On the left, the data points represent different values of $\hat{p}_{0}$ for $\varphi_{0}=0,1,2$, and 3 , in black (dots), dashed blue (squares), dotted red (diamonds) and dot/dash purple (triangles) respectively. On the right, the data points represent different values of $\varphi_{0}$ for $\hat{p}_{0}=0.75,1,1.5$ and 2 , in black (dots), dashed blue (squares), dotted red (diamonds) and dot/dash purple (triangles) respectively.

black hole varies as a function of temperature at fixed $r_{+}=1$ for different values of initial data. The two plots show set of data for $S(T)$ having fixed $\varphi_{0}$ and varying $\hat{p}_{0}$ (left) or data for fixed $\hat{p}_{0}$ and varying $\varphi_{0}$ (right). In the left plot, we see that just as varying $\hat{p}_{0}$ at fixed $\varphi_{0}$ has a much more uniform effect on temperature, so varying $\hat{p}_{0}$ at fixed $\varphi_{0}$ has a somewhat more consistent effect on entropy, although curiously the entropy generally drops as we increase the temperature. For $\varphi_{0}=0$ however, the entropy remains fairly constant. Since we have fixed $r_{+}=1$, we would not necessarily expect the entropy to vary hugely with $\hat{p}_{0}$, at least by analogy with the Reissner-Nordstrom solution. 
When exploring the $S(T)$ plot for varying $\varphi_{0}$ however, the picture becomes much more interesting. We would expect entropy to vary much more strongly with $\varphi_{0}$ at fixed $r_{+}$, since we have seen from our eigenvalue analysis of the perturbations that the two "scalar" modes, the dilaton and the internal breather $H$, are very much coupled by the equations of motion. We therefore expect that altering $\varphi_{0}$ will alter $H\left(r_{+}\right)$and hence the entropy to a much greater extent, and this is indeed what we see. However, what is interesting is the modulating behaviour of both the temperature and entropy as a function of $\varphi_{0}$. We see that at a given temperature and $\hat{p}_{0}$, there are two possible values for the scalar charge, one with higher entropy that the other. Although it is not entirely clear from the plot, the curves have $\varphi_{0}$ increasing in a clockwise direction, hence it is the black hole with lower $\varphi_{0}$ that is entropically preferred. This indicates that these black holes will likely have scalar instabilities, perhaps shedding scalar charge to increase their overall horizon area. How this is consistent with the usual concept of a black hole accreting to increase its area might prove an interesting investigation.

Finally, we should comment on the impact of varying $r_{+}$: Because of the scaling symmetry present in the equations of motion, all of the numerically computed fields are dependent on $r / r_{+}$, and thus simply stretch with $r_{+}$. In particular, the horizon value of $H$ does not change with $r_{+}$, and the derivatives of $F$ and $D$ just scale as $1 / r_{+}$. Thus, the entropy scales as $r_{+}^{2}$, independent of the value of $z$, and the temperature as $r_{+}^{z}$. (Of course, the entropy and temperature vary with the initial data of the charges as we have seen.) The variation of entropy with temperature is therefore explicitly the expected relation $S \propto T^{2 / z}$ for a field theory in flat $2+1$ dimensions.

\section{Conclusions}

In this paper we have built string black hole configurations that asymptote Lifshitz geometries for general dynamical exponents $z>1$. We began by finding these solutions in a supergravity theory that corresponds to a consistent truncation of massive type IIA string theory, namely the maximal gauged six dimensional supergravity of Romans, and then uplifted them to massive type IIA supergravity. They are related to asymptotically adS black hole solutions in the same supergravity, also studied here, and extensions of the pure Lifshitz geometries found in [17].

Our supergravity theory has rich field content, and yet allows simple pure $\operatorname{adS}_{4} \times \mathrm{H}_{2}$ and $\mathrm{Li}_{4} \times H_{2}$ compactifications. By expressing the full set of equations in the form of an autonomous dynamical system, we studied perturbations around these exact solutions, determining analytically all the possible asymptotic behaviours of static, planar adS and Lifshitz black holes for our theory. We found that various asymptotics are allowed, depending on which fields are switched on and on the values of the parameters involved, in particular the dynamical exponent $z$.

Starting with the adS case, one exact analytic solution is of course known: adSSchwarzschild. By studying this background, and exciting the supergravity two-form potential $B_{2}$ and dilaton in the probe limit, we acquired insight into the properties of adS black holes. In particular, apart from the horizon size, the black holes are characterized 
by two quantities, $\varphi_{0}$ and $p_{0}$, which we are motivated to call scalar and $B$-charge. After exploring the adS case, we applied the intuition gained from it to analyze Lifshitz configurations, for arbitrary dynamical exponent $z>1$. In fact, a non-trivial scalar and $B$-charge is a necessary ingredient for obtaining asymptotically Lifshitz geometries. Although the notion of a charged black hole in the Lifshitz case is less straightforward than for adS, since any Lifshitz configuration is always characterized by the presence of all fields, we thus determined the independent, tunable quantities characterizing Lifshitz black holes.

We were able to formulate a precise analytical understanding as to how asymptotically Lifshitz configurations reduce to adS ones, in the limit $z \rightarrow 1$, which could be helpful in the future for comparing predictions of field theory duals of adS and Lifshitz theories. Then, we studied numerically how the asymptotics match black hole horizons at finite values of the radial coordinates, computing two-parameter families of black holes, for both adS and Lifshitz asymptotics.

One common feature displayed by the Lifshitz black holes is the presence of a nonmonotonicity in the time and radial metric potentials, $F$ and $D$, particularly well illustrated in figure 9 . This peaking of the Newtonian potential becomes extremely marked at lower $\varphi_{0}$ and $\hat{p}_{0}$, with $F$ peaking at over 50 or higher. Although it might appear as if a large peak in $F$ could provide a barrier to approaching the event horizon, in fact this is outweighed by the strong Lifshitz $r^{2 z}$ warp factor, and particles are always pulled into the black hole.

As well as studying the field profiles for the black hole solutions, and how they vary with $B$-charge, scalar charge and dynamical exponent $z$, we initiated a study of the thermodynamics of our black holes. We find the $B$-charge to play an analagous role to the charge in the Reissner-Nordström black hole, with temperature decreasing as the charge increases, however, the zero charge limit is more subtle for the Lifshitz black holes. Meanwhile, adS/Lifshitz black holes exist only for a finite range of scalar charge, due to the Liouville type scalar potential, and in general for a given temperature there are two possible scalar charges, perhaps surprisingly the smaller one being entropically preferred. The interplay between the scalar and $B$-charge is also interesting, in particular a high scalar charge reduces the amount of possible $B$-charge.

Since we are interested in holographic condensed matter applications, we have focussed on planar black hole geometries, corresponding to a finite temperature boundary theory in flat $2+1$ dimensions. In simpler models, with pure geometry planar black holes, the scaling symmetry renders black holes with different horizon sizes, and thus temperatures, physically equivalent. However, in our supergravity construction the necessary, additional scales of $B$-charge and scalar charge introduce the possibility of phase transitions. It would be interesting to investigate this possibility further, as well as their interpretation in the dual field theory. ${ }^{4}$ Moreover, the extra fields present in the supergravity theory, which we have chosen not to activate, represent other possibilities for interesting black hole charges and phase transitions.

Another aspect that deserves further thought would be the brane interpretation of our

\footnotetext{
${ }^{4}$ Phase transitions in a phenomenological model that strongly resembles our stringy supergravity setup have been studied very recently in [50].
} 
type IIA configuration. In the end, we hope that having explicit string theory embeddings of Lifshitz black hole geometries will help to develop the holographic description of interesting anisotropic condensed matter systems.

\section{Acknowledgments}

We would like to thank Harry Braviner, Anton Faedo and Simon Ross for discussions. LB was supported by an STFC studentship. RG is supported in part by STFC (Consolidated Grant ST/J000426/1), in part by the Wolfson Foundation and Royal Society, and in part by Perimeter Institute for Theoretical Physics. Research at Perimeter Institute is supported by the Government of Canada through Industry Canada and by the Province of Ontario through the Ministry of Economic Development and Innovation. SLP is funded by the Deutsche Forschungsgemeinschaft (DFG) inside the "Graduiertenkolleg GRK 1463". GT is supported by an STFC Advanced Fellowship ST/H005498/1. We would also like to thank the Aspen Center for Physics for hospitality via the Working Group Program.

\section{A The autonomous dynamical system}

In this appendix we reformulate the system of supergravity equations of motion as an autonomous dynamical system. This requires the use of a different gauge to the numerical work, however the translation between the gauges is straightforward. Choosing $d=-\phi / \sqrt{2}-\ln L g$, in the general planar metric (2.13), ensures that only negative exponents of $\phi$ appear in the equations of motion. Then, defining

$$
\begin{aligned}
& X_{1}=\sqrt{\frac{m}{g}} e^{-\sqrt{2} \phi}, \quad X_{2}=\frac{q}{g} e^{-\sqrt{2} \phi-2 h}, \quad X_{3}=\sqrt{2} \phi^{\prime}, \quad X_{4}=2 f^{\prime}, \\
& X_{5}=2 h^{\prime}+\sqrt{2} \phi^{\prime}, \quad X_{6}=2 c^{\prime}, \quad X_{7}=e^{-2 c+\sqrt{2} \phi} P, \quad X_{8}=e^{-2 c+\sqrt{2} \phi} P^{\prime}
\end{aligned}
$$

yields the dynamical system

$$
\begin{aligned}
X_{1}^{\prime}= & -X_{1} X_{3} \quad X_{2}^{\prime}=-X_{2} X_{5} \\
X_{3}^{\prime}= & -X_{2}^{2}+X_{7}^{2}\left(X_{2}^{2}-\frac{X_{1}^{4}}{4}\right)-\frac{1}{4}+X_{1}^{2}-\frac{3}{4} X_{1}^{4} \\
& -X_{3}\left(\frac{X_{4}-X_{3}}{2}+X_{5}+X_{6}\right)+\frac{X_{8}^{2}}{2} \\
X_{4}^{\prime}= & X_{2}^{2}+X_{7}^{2}\left(3 X_{2}^{2}+\frac{X_{1}^{4}}{4}\right)+\frac{1}{4}+X_{1}^{2}-\frac{X_{1}^{4}}{4} \\
& -X_{4}\left(\frac{X_{4}-X_{3}}{2}+X_{5}+X_{6}\right)+\frac{X_{8}^{2}}{2} \\
X_{5}^{\prime}= & \left(X_{7}^{2}-1\right)\left(2 X_{2}^{2}+\frac{X_{1}^{4}}{2}\right)-\frac{1}{2}+\frac{X_{8}^{2}}{2}+X_{4} X_{6}+\frac{X_{6}^{2}}{2} \\
& +\left(X_{5}-2 X_{3}\right)\left(\frac{X_{4}}{2}+X_{6}\right)-\frac{X_{3}^{2}+X_{3} X_{5}+X_{5}^{2}}{2}
\end{aligned}
$$




$$
\begin{aligned}
X_{6}^{\prime}= & X_{2}^{2}-X_{7}^{2}\left(X_{2}^{2}+\frac{3 X_{1}^{4}}{4}\right)+\frac{1}{4}+X_{1}^{2}-\frac{X_{1}^{4}}{4} \\
& -X_{6}\left(\frac{X_{4}-X_{3}}{2}+X_{5}+X_{6}\right)-\frac{X_{8}^{2}}{2} \\
X_{7}^{\prime}= & X_{8}-X_{6} X_{7}+X_{3} X_{7} \\
X_{8}^{\prime}= & -X_{8}\left(\frac{1}{2} X_{4}+X_{5}+\frac{X_{3}}{2}\right)+X_{7}\left[X_{1}^{4}+4 X_{2}^{2}\right]
\end{aligned}
$$

in which the solution lies in an invariant $7 \mathrm{D}$ submanifold described by the constraint

$$
\begin{aligned}
& 2 X_{4}\left(X_{6}+X_{5}-X_{3}\right)+4\left(X_{5}-X_{3}\right) X_{6}+X_{6}^{2}+X_{5}^{2}-2 X_{5} X_{3}-X_{3}^{2}-X_{8}^{2} \\
& \quad=4 \frac{K}{q g} X_{2}-4 X_{2}^{2}-X_{7}^{2}\left(4 X_{2}^{2}+X_{1}^{4}\right)+\left(1+4 X_{1}^{2}-X_{1}^{4}\right) .
\end{aligned}
$$

For a critical point, (A.2) implies $X_{3}=X_{5}=0$, and (A.7), (A.8) give:

$$
\begin{aligned}
X_{8} & =X_{6} X_{7} \\
X_{4} X_{8} & =2 X_{7}\left[X_{1}^{4}+4 X_{2}^{2}\right]
\end{aligned}
$$

These are solved by either $X_{7}=X_{8}=0$, or

$$
X_{4} X_{6}=2 X_{1}^{4}+8 X_{2}^{2} \text {. }
$$

We can distinguish two cases:

- Case 1: adS

In this case $X_{7}=X_{8}=0$. Solving (A.3)-(A.6) gives

$$
\begin{aligned}
X_{1}^{2} & =1-\sqrt{1-\frac{3}{2} X_{6}^{2}} \\
X_{2}^{2} & =\frac{9}{8} X_{6}^{2}-\frac{3}{4}+\frac{1}{2} \sqrt{1-\frac{3}{2} X_{6}^{2}} \\
X_{4} & =X_{6}
\end{aligned}
$$

with the constraint

$$
\frac{2 X_{2}}{q g}=3\left(1-X_{6}^{2}\right)-2 \sqrt{1-\frac{3}{2} X_{6}^{2}}
$$

selecting two values for $X_{6}$ for each charge. In terms of the supergravity parameters, $\hat{g}=2 / X_{6}, \hat{m}=2 x_{1}^{2} / X_{6}$. To get the solution in the original area gauge, note that $r=e^{c}=e^{X_{6} \rho / 2}$. These critical points form a curve of adS solutions in the phase space, with the curve intersecting the invariant submanifold at two points in general.

To analyze the nature of the critical points, one takes small perturbations, and finds the eigenvalues and eigenvectors of the perturbation operator matrix given by $\delta X_{\alpha}^{\prime}=$ $M_{\alpha \beta} X_{\beta}$, i.e. $M v^{(i)}=\lambda_{i} v^{(i)}$. There will always be one zero eigenvalue to this matrix, corresponding to moving along the adS solution curve. The remaining 7 th order polynomial can be factorized, yielding the eigenvalues plotted in figure 2, although note that to obtain figure 2 , we have transformed our coordinates to the area gauge to get the $\Delta_{i}$ to correspond to the $r$ fall-off exponents: $\Delta_{i}=2 \lambda_{i} / X_{6}=\hat{g} \lambda_{i}$. 
- Case 2: Lifshitz

Here $X_{8}=X_{6} X_{7}$, and (A.12) holds, and solving the remaining equations gives:

$$
\begin{aligned}
& X_{4}=X_{6}\left(1+X_{7}^{2}\right)=\sqrt{\frac{2\left(1+X_{7}^{2}\right)}{\left(5+X_{7}^{2}\right)}} \\
& X_{1}^{2}=\frac{\left(5+X_{7}^{2}\right) \mp \sqrt{2\left(5+X_{7}^{2}\right)}}{\left(1+X_{7}^{2}\right)\left(5+X_{7}^{2}\right)} \\
& X_{2}^{2}=\frac{\left(X_{7}^{2}+3\right)\left(X_{7}^{2}-2\right) \pm 2 \sqrt{2\left(5+X_{7}^{2}\right)}}{4\left(5+X_{7}^{2}\right)\left(1+X_{7}^{2}\right)^{2}} .
\end{aligned}
$$

For $X_{7}^{2}<\sqrt{2}-(1+\sqrt{17+4 \sqrt{2}}) / 2 \simeq 3.3$, in (A.19) only the upper branch choice gives a real solution for $X_{2}$. Finally, the constraint determines the charge:

$$
q g=\frac{\sqrt{\left(X_{7}^{2}+5\right)\left(\left(X_{7}^{2}+3\right)\left(X_{7}^{2}-2\right) \pm 2 \sqrt{2\left(X_{7}^{2}+5\right)}\right)}}{3\left(X_{7}^{2}+3\right) \mp 2 \sqrt{2\left(X_{7}^{2}+5\right)}} .
$$

Clearly $X_{7}^{2}=z-1$, and this is equivalent to the exact solution (2.11) in the area gauge.

The analysis of the perturbations around the Lifshitz critical points, although conceptually identical to adS, is algebraically more involved. The eigenvalues pair around $-(z+2) / 2$ (in area gauge) with the variance given by the square root of the solution of a cubic equation. While this can be written in closed form, it is a rather long and unilluminating expression. Figure 4 shows a plot of the eigenvalues renormalized for a $r$ fall-off: $\Delta_{i}=\sqrt{2 z(4+z)} \lambda_{i}$. All of the eigenvectors of the perturbation operator have nonzero components in the vector, scalar and geometry directions.

\section{B Exact Lifshitz solutions}

So far in the literature it has been possible to obtain exact Lifshitz black hole (LiBH) solutions only in some phenomenological models, where the matter content is engineered to support the desired geometry. In this appendix we give a brief account of these approaches, and extend them to dilatonic models, which may be more easily embedded into supergravity and string theory. We then show that such simple analytical solutions cannot be found in the Romans' 6D supergravity that is the main subject of this paper.

Analytic LiBH solutions have been found for essentially two types of 4D Einstein gravity systems (see also [37-44] for other possible extensions). The first $(\Lambda \mathcal{A} A m)$ contains, besides gravity, a cosmological constant, a massless abelian gauge field $\mathcal{F}_{2}$ and massive abelian gauge field $F_{2}$ with mass $m[26]$. In $4 \mathrm{D}$, the massive gauge field is equivalent to a 2-form $B_{2}$ and a massless gauge field $F_{2}$ with non trivial Chern-Simons terms $F_{2} \wedge B_{2}$, as studied in [7]. The second system $(\Lambda \mathcal{A} \phi)$ consists of gravity, a cosmological constant, a number of massless abelian gauge fields $\mathcal{F}_{2}^{i}$ and a massless scalar field $\phi$ with dilaton-like couplings to the gauge sector $[7,27]$. An obstruction to straightfowardly embedding these setups into supergravity and string theory is their absence of a genuine dilaton field. For 
example, many supergravity theories, like Romans' 6D supergravity, necessarily contain a dilaton field in the supergravity multiplet. Indeed, it seems unlikely that the $\Lambda \mathcal{A} \phi$ system could be embedded in string theory, without generalizing the cosmological constant to a genuine dilatonic potential. It is then easy to check that in the presence of a non-trivial dilaton potential, the dilaton equation of motion prevents the Lifshitz asymptotics. This can be observed from the general solutions discussed in [52].

The $\Lambda \mathcal{A} A m$ system is more interesting. Building on $[13-16,51]$, a similar model, though without the massless vector and with two additional dilatonic/radion scalars, was obtained in [45] via a consistent massive truncation of Type IIB on an arbitrary Einstein space times $S^{1}$, and used to derive numerical stringy LiBHs. Here, we generalise the system to a general dilatonic theory, with generic dilaton-matter couplings and dilaton potential, and search for analytical LiBHs.

\section{B.1 4D LiBHs with constant dilaton}

We consider the four dimensional case, which is sufficient to illustrate our strategy. As well as massless and massive abelian gauge fields, we add a dilaton field $\phi$ with couplings $\lambda, \sigma$ to the gauge fields and a general potential $V(\phi)$. In this section we follow closely the discussions in $[26,27]$, and assume their mostly plus metric signature conventions.

We take the general action:

$$
S=\frac{1}{\kappa_{4}^{2}} \int d^{4} x \sqrt{g}\left[R-\frac{1}{2}(\partial \phi)^{2}-\frac{e^{-\lambda \phi}}{4} F_{2}^{2}-\frac{e^{-\lambda \phi} m^{2}}{2} A_{1}^{2}-\frac{e^{-\sigma \phi}}{4} \mathcal{F}_{2}^{2}-V(\phi)\right]
$$

with corresponding equations of motion:

$$
\begin{aligned}
& \partial_{\mu}\left[\sqrt{-g} e^{-\lambda \phi} F^{\mu \nu}\right]=\sqrt{-g} e^{-\lambda \phi} m^{2} A^{\nu} \\
& \partial_{\mu}\left[\sqrt{-g} e^{-\sigma \phi} \mathcal{F}^{\mu \nu}\right]=0 \\
& \frac{1}{\sqrt{-g}} \partial_{\mu}\left[\sqrt{-g} g^{\mu \nu} \partial_{\nu} \phi\right]=\frac{\partial V}{\partial \phi}-\frac{\lambda e^{-\lambda \phi}}{4} F^{2}-\frac{\lambda m^{2} e^{-\lambda \phi}}{2} A^{2}-\frac{\sigma e^{-\sigma \phi}}{4} \mathcal{F}^{2} \\
& R_{\mu \nu}=\frac{1}{2} \partial_{\mu} \phi \partial_{\nu} \phi+\frac{V}{2} g_{\mu \nu}+\frac{e^{-\lambda \phi}}{4}\left[2 F_{\mu}^{\lambda} F_{\mu \lambda}-\frac{g_{\mu \nu}}{2} F^{2}+2 m^{2} A_{\mu} A_{\nu}\right] \\
& +\frac{e^{-\sigma \phi}}{4}\left[2 \mathcal{F}_{\mu}^{\lambda} \mathcal{F}_{\mu \lambda}-\frac{g_{\mu \nu}}{2} \mathcal{F}^{2}\right] \text {. }
\end{aligned}
$$

Consider now the metric Ansatz

$$
d s^{2}=-r^{2 z} h(r) d t^{2}+\frac{d r^{2}}{r^{2} h(r)}+r^{2} d x_{i}^{2}
$$

sourced by $\mathcal{F}_{r t}, \mathcal{F}_{x_{1} x_{2}}$ and $F_{r t}$, plus a constant dilaton field $\phi=$ const. Solving the field equations for the forms gives:

$$
\begin{aligned}
& \mathcal{F}_{r t}=Q_{1} r^{z-3}, \quad \mathcal{F}_{x_{1} x_{2}}=Q_{2} \\
& \partial_{r}\left[r^{3-z} \partial_{r} A_{t}\right]=\frac{m^{2} A_{t}}{r^{z-1} h(r)} .
\end{aligned}
$$


From the Einstein equations $R_{t}^{t}-R_{r}^{r}$, we obtain the solution for the gauge field:

$$
A_{t}= \pm \frac{2}{m} e^{\lambda \phi / 2} \sqrt{z-1} r^{z} h,
$$

and then using (B.8) we find the metric function:

$$
h=\frac{m^{2}}{2 z}+\frac{C_{1}}{(z-2)} \frac{1}{r^{2}}+\frac{C_{2}}{r^{z}},
$$

for $z \neq 2$, and an analogous expression for $z=2$.

Now the dilaton and remaining Einstein equations can be solved provided that the following constraints among the parameters are satisfied:

$$
\begin{aligned}
z & =4 \\
C_{1} & =0 \\
-C_{2} & =\frac{1}{8} e^{-\sigma \phi}\left(Q_{2}^{2}+Q_{1}^{2}\right) \quad \Rightarrow \quad C_{2}<0 \\
-\frac{m^{2}}{4 z}[2(z+2)+z(z-1)] & =\frac{V}{2} \\
2 \lambda(z-1) C_{2} & =\frac{\sigma}{2} e^{-\sigma \phi}\left(Q_{2}^{2}-Q_{1}^{2}\right) \\
\lambda(z-1) m^{2}\left(\frac{1}{2}+\frac{1}{z}\right) & =-\frac{\partial V}{\partial \phi} .
\end{aligned}
$$

The first four constraints above correspond to those in [26] where there is no dilaton, and the dilaton adds two more. In the end there are four non-trivial constraints on the four solution parameters $C_{2}, Q_{1}, Q_{2}$ and $\phi$, plus the mass parameter $m$ and any gauge couplings that appear in $V(\phi)$. So, provided that these constraints can be solved consistently, we can avoid tuning the dilaton couplings $\lambda, \sigma$.

\section{B.2 6D Romans' LiBH with constant dilaton?}

Romans' 6D supergravity has a strong resemblance to the dilatonic theory we just discussed, or its Chern-Simons equivalent. Besides having two extra dimensions, the main difference is that the 2-form potential has not only a Chern-Simons term but also a mass term. Nevertheless, it is straightforward to apply the above strategy to search for LiBH solutions with constant dilaton in Romans' supergravity. These solutions would be orthogonal to the ones we discuss in the main text, since they involve turning on additional fields.

Indeed, whereas in the main text we activated only one of the gauge fields, we now add a non-trivial configuration for the second gauge field, taking the Ansatz (2.9) plus:

$$
\mathcal{F}_{r t}=Q_{1} r^{z-3} \quad \mathcal{F}_{x_{1} x_{2}}=Q_{2} .
$$

The expression for the metric function that follows is:

$$
h(r)=\frac{\mathrm{m} Q_{2} e^{-2 \sqrt{2} \phi_{0}}}{(z-2) \sqrt{z-1}} \frac{1}{r^{2}}+\frac{C_{1}}{r^{z}}+\frac{L^{2}}{2 z}\left[\mathrm{~m}^{2} e^{-3 \sqrt{2} \phi_{0}}+4 \frac{q^{2}}{a^{4}} e^{-\sqrt{2} \phi_{0}}\right],
$$


for $z \neq 2$, and an analogous expression for $z=2$. Unfortunately, the constraints coming from the field equations can only be solved by a pure Lifshitz configuration, with $Q_{1}=$ $0=Q_{2}$ and $C_{1}=0$, and the relations (2.11). Thus we cannot construct a simple analytic solution via this method.

Open Access. This article is distributed under the terms of the Creative Commons Attribution License which permits any use, distribution and reproduction in any medium, provided the original author(s) and source are credited.

\section{References}

[1] J.M. Maldacena, The large- $N$ limit of superconformal field theories and supergravity, Adv. Theor. Math. Phys. 2 (1998) 231 [Int. J. Theor. Phys. 38 (1999) 1133] [hep-th/9711200] [INSPIRE].

[2] S.A. Hartnoll, Lectures on holographic methods for condensed matter physics, Class. Quant. Grav. 26 (2009) 224002 [arXiv:0903.3246] [INSPIRE].

[3] S. Sachdev, Condensed matter and AdS/CFT, arXiv:1002.2947 [INSPIRE].

[4] D. Son, Toward an AdS/cold atoms correspondence: a geometric realization of the Schrödinger symmetry, Phys. Rev. D 78 (2008) 046003 [arXiv: 0804.3972] [INSPIRE].

[5] K. Balasubramanian and J. McGreevy, Gravity duals for non-relativistic CFTs, Phys. Rev. Lett. 101 (2008) 061601 [arXiv:0804.4053] [INSPIRE].

[6] S. Kachru, X. Liu and M. Mulligan, Gravity duals of Lifshitz-like fixed points, Phys. Rev. D 78 (2008) 106005 [arXiv:0808.1725] [INSPIRE].

[7] M. Taylor, Non-relativistic holography, arXiv:0812.0530 [INSPIRE].

[8] K. Copsey and R. Mann, Pathologies in asymptotically Lifshitz spacetimes, JHEP 03 (2011) 039 [arXiv:1011.3502] [INSPIRE].

[9] G.T. Horowitz and B. Way, Lifshitz singularities, Phys. Rev. D 85 (2012) 046008 [arXiv: 1111.1243] [INSPIRE].

[10] S.A. Hartnoll, J. Polchinski, E. Silverstein and D. Tong, Towards strange metallic holography, JHEP 04 (2010) 120 [arXiv:0912.1061] [INSPIRE].

[11] J. Blaaback, U.H. Danielsson and T. Van Riet, Lifshitz backgrounds from 10d supergravity, JHEP 02 (2010) 095 [arXiv: 1001.4945] [INSPIRE].

[12] K. Balasubramanian and K. Narayan, Lifshitz spacetimes from AdS null and cosmological solutions, JHEP 08 (2010) 014 [arXiv: 1005.3291] [INSPIRE].

[13] A. Donos and J.P. Gauntlett, Lifshitz solutions of $D=10$ and $D=11$ supergravity, JHEP 12 (2010) 002 [arXiv: 1008.2062] [INSPIRE].

[14] A. Donos, J.P. Gauntlett, N. Kim and O. Varela, Wrapped M5-branes, consistent truncations and AdS/CMT, JHEP 12 (2010) 003 [arXiv: 1009.3805] [INSPIRE].

[15] D. Cassani and A.F. Faedo, Constructing Lifshitz solutions from AdS, JHEP 05 (2011) 013 [arXiv: 1102.5344] [INSPIRE].

[16] W. Chemissany and J. Hartong, From D3-branes to Lifshitz space-times, Class. Quant. Grav. 28 (2011) 195011 [arXiv:1105.0612] [InSPIRE]. 
[17] R. Gregory, S.L. Parameswaran, G. Tasinato and I. Zavala, Lifshitz solutions in supergravity and string theory, JHEP 12 (2010) 047 [arXiv: 1009.3445] [INSPIRE].

[18] L. Romans, The F(4) gauged supergravity in six-dimensions, Nucl. Phys. B 269 (1986) 691 [INSPIRE].

[19] L. Romans, Gauged $N=4$ supergravities in five-dimensions and their magnetovac backgrounds, Nucl. Phys. B 267 (1986) 433 [INSPIRE].

[20] M. Cvetič, H. Lü and C. Pope, Gauged six-dimensional supergravity from massive type IIA, Phys. Rev. Lett. 83 (1999) 5226 [hep-th/9906221] [INSPIRE].

[21] H. Lü, C. Pope and T.A. Tran, Five-dimensional $N=4, \mathrm{SU}(2) \times \mathrm{U}(1)$ gauged supergravity from type IIB, Phys. Lett. B 475 (2000) 261 [hep-th/9909203] [INSPIRE].

[22] M. Cvetič, H. Lü and C. Pope, Consistent warped space Kaluza-Klein reductions, half maximal gauged supergravities and CPn constructions, Nucl. Phys. B 597 (2001) 172 [hep-th/0007109] [INSPIRE].

[23] H. Singh, Galilean anti-de-Sitter spacetime in Romans theory, Phys. Lett. B 682 (2009) 225 [arXiv:0909.1692] [INSPIRE].

[24] N. Halmagyi, M. Petrini and A. Zaffaroni, Non-relativistic solutions of $N=2$ gauged supergravity, JHEP 08 (2011) 041 [arXiv:1102.5740] [INSPIRE].

[25] U.H. Danielsson and L. Thorlacius, Black holes in asymptotically Lifshitz spacetime, JHEP 03 (2009) 070 [arXiv: 0812.5088] [INSPIRE].

[26] D.-W. Pang, On charged Lifshitz black holes, JHEP 01 (2010) 116 [arXiv:0911.2777] [INSPIRE].

[27] J. Tarrío and S. Vandoren, Black holes and black branes in Lifshitz spacetimes, JHEP 09 (2011) 017 [arXiv:1105.6335] [INSPIRE].

[28] R.B. Mann, Lifshitz topological black holes, JHEP 06 (2009) 075 [arXiv:0905.1136] [INSPIRE].

[29] G. Bertoldi, B.A. Burrington and A. Peet, Black holes in asymptotically Lifshitz spacetimes with arbitrary critical exponent, Phys. Rev. D 80 (2009) 126003 [arXiv:0905.3183] [INSPIRE].

[30] G. Bertoldi, B.A. Burrington and A.W. Peet, Thermodynamics of black branes in asymptotically Lifshitz spacetimes, Phys. Rev. D 80 (2009) 126004 [arXiv:0907.4755] [INSPIRE].

[31] E. Brynjolfsson, U. Danielsson, L. Thorlacius and T. Zingg, Holographic Superconductors with Lifshitz Scaling, J. Phys. A 43 (2010) 065401 [arXiv:0908.2611] [InSPIRE].

[32] M.C. Cheng, S.A. Hartnoll and C.A. Keeler, Deformations of Lifshitz holography, JHEP 03 (2010) 062 [arXiv:0912.2784] [INSPIRE].

[33] E. Ayon-Beato, A. Garbarz, G. Giribet and M. Hassaine, Analytic Lifshitz black holes in higher dimensions, JHEP 04 (2010) 030 [arXiv:1001.2361] [INSPIRE].

[34] G. Bertoldi, B.A. Burrington and A.W. Peet, Thermal behavior of charged dilatonic black branes in AdS and UV completions of Lifshitz-like geometries,

Phys. Rev. D 82 (2010) 106013 [arXiv:1007.1464] [INSPIRE]. 
[35] G. Bertoldi, B.A. Burrington, A.W. Peet and I.G. Zadeh, Lifshitz-like black brane thermodynamics in higher dimensions, Phys. Rev. D 83 (2011) 126006 [arXiv:1101.1980] [INSPIRE].

[36] M. Dehghani, R. Mann and R. Pourhasan, Charged Lifshitz black holes, Phys. Rev. D 84 (2011) 046002 [arXiv: 1102.0578] [INSPIRE].

[37] R.B. Mann, Lifshitz topological black holes, JHEP 06 (2009) 075 [arXiv:0905.1136] [INSPIRE].

[38] K. Balasubramanian and J. McGreevy, An analytic Lifshitz black hole, Phys. Rev. D 80 (2009) 104039 [arXiv:0909.0263] [INSPIRE].

[39] E. Ayon-Beato, A. Garbarz, G. Giribet and M. Hassaine, Lifshitz black hole in three dimensions, Phys. Rev. D 80 (2009) 104029 [arXiv:0909.1347] [InSPIRE].

[40] R.-G. Cai, Y. Liu and Y.-W. Sun, A Lifshitz black hole in four dimensional $R^{2}$ gravity, JHEP 10 (2009) 080 [arXiv:0909.2807] [INSPIRE].

[41] M. Dehghani and R.B. Mann, Lovelock-Lifshitz black holes, JHEP 07 (2010) 019 [arXiv: 1004 . 4397] [INSPIRE].

[42] W. Brenna, M. Dehghani and R. Mann, Quasi-topological Lifshitz black holes, Phys. Rev. D 84 (2011) 024012 [arXiv:1101.3476] [INSPIRE].

[43] H. Maeda and G. Giribet, Lifshitz black holes in Brans-Dicke theory, JHEP 11 (2011) 015 [arXiv:1105.1331] [INSPIRE].

[44] J. Matulich and R. Troncoso, Asymptotically Lifshitz wormholes and black holes for Lovelock gravity in vacuum, JHEP 10 (2011) 118 [arXiv:1107.5568] [INSPIRE].

[45] I. Amado and A.F. Faedo, Lifshitz black holes in string theory, JHEP 07 (2011) 004 [arXiv: 1105.4862] [INSPIRE].

[46] J.M. Maldacena and C. Núñez, Supergravity description of field theories on curved manifolds and a no go theorem, Int. J. Mod. Phys. A 16 (2001) 822 [hep-th/0007018] [InSPIRE].

[47] H. Braviner, R. Gregory and S.F. Ross, Flows involving Lifshitz solutions, Class. Quant. Grav. 28 (2011) 225028 [arXiv:1108.3067] [INSPIRE].

[48] P. Breitenlohner and D.Z. Freedman, Positive Energy in anti-de Sitter Backgrounds and Gauged Extended Supergravity, Phys. Lett. B 115 (1982) 197 [InSPIRE].

[49] P. Breitenlohner and D.Z. Freedman, Stability in gauged extended supergravity, Annals Phys. 144 (1982) 249 [INSPIRE].

[50] E. D'Hoker and P. Kraus, Charge expulsion from black brane horizons and holographic quantum criticality in the plane, arXiv:1202.2085 [inSPIRE].

[51] J. Maldacena, D. Martelli and Y. Tachikawa, Comments on string theory backgrounds with non-relativistic conformal symmetry, JHEP 10 (2008) 072 [arXiv:0807.1100] [INSPIRE].

[52] C. Burgess, C. Núñez, F. Quevedo, G. Tasinato and I. Zavala, General brane geometries from scalar potentials: gauged supergravities and accelerating universes, JHEP 08 (2003) 056 [hep-th/0305211] [INSPIRE]. 